\title{
1 Early Detection of Tau Pathology
}

3 Parag Parekh ${ }^{1}$, Andrew Badachhape ${ }^{1}$, Qingshan $\mathrm{Mu}^{1}$, Rohan Bhavane ${ }^{2}$, Mayank Srivastava ${ }^{2}$, Laxman

4 Devkota ${ }^{1}$, Jason Eriksen ${ }^{3}$, Eric Tanifum ${ }^{4}$, Ketan Ghaghada ${ }^{4}$, and Ananth Annapragada ${ }^{4, *}$

$5{ }^{1}$ Baylor College of Medicine, Houston, TX, USA, ${ }^{2}$ Texas Children's Hospital, Houston, TX, USA, ${ }^{3}$ College of

6 Pharmacy, University of Houston, Houston, TX, USA, ${ }^{4}$ Texas Children's Hospital/Baylor College of

7 Medicine, Houston, TX, USA

$8 \quad$ Email: annaprag@bcm.edu/avannapr@texaschildrens.org

\section{Abstract}

While a definitive Alzheimer's disease (AD) diagnosis remains a post-mortem exercise, the ATN Research Framework proposed by the National Institute on Aging and the Alzheimer's Association utilizes a score representing the presence of amyloid deposits (A), tau deposits $(T)$ and neuronal degeneration markers $(\mathrm{N})$, with $\mathrm{A}+\mathrm{T}+$ necessary for a positive diagnosis. Current detection of tau pathology lags amyloid detection by years and by the time both markers are detected the disease is fairly advanced. We describe the development of a new generation of molecular imaging probes for in vivo detection of cells undergoing abnormal phosphorylation representing the initial stages of pTau pathology, potentially enabling a very early stage diagnosis of AD. We describe a novel nanoparticle formulation that binds such abnormally phosphorylating cells in a mouse model of tau pathology, enabling in vivo visualization of the hyperphosphorylative state by magnetic resonance imaging. Our results demonstrate the potential of this novel platform to identify a correlative marker signifying the development of future tau pathology, and has implications for early-stage diagnosis of Alzheimer's disease.

\section{Introduction}

The microtubule associated protein tau, coded for by the MAPT gene, is abundant in the brain and is present in neurons, glia and other cell types. Tau shows immense diversity. It is expressed in six isoforms and has a vast array of post-translational modifications, including glycosylation, glycation, nitration, ubiquitination and more than 80 phosphorylation sites expanding the complexity of its role in health and disease ${ }^{1,2}$. A definitive feature of many neurodegenerative diseases including $A D$, frontotemporal lobar degeneration (FTLD), 
brains. The transition from physiological soluble tau is primarily associated with changes in its phosphorylation state leading to oligomeric tau ${ }^{7}$, then tau fibrils known as paired helical fragments (PHF) that collectively form characteristic neurofibrillary tangles (NFT). The tau aggregates are then capable of "infecting" a healthy cell inducing further misfolding, aggregation, and neuro-toxicity 8,9 . Studies of intercellular propagation demonstrate passage through an extracellular phase that progresses throughout the brain ${ }^{10,11}$.

The research framework proposed for precise diagnosis of AD by the National Institute of AgingAlzheimer's Association (NIA-AA) categorizes extracellular deposits of amyloid beta (A), presence of intraneuronal hyperphosphorylated tau $(\mathrm{T})$ and markers of neurodegeneration or neuronal injury $(\mathrm{N})$. Each biomarker is scored either positive or negative ${ }^{12}$. To be on the AD continuum, $A+$ (Amyloid positive) is required, while a positive diagnosis of $A D$ requires $A+$ and $T+$. Biomarker detection can be by i) positron emission tomography (PET) imaging of amyloid and tau or, ii) cerebrospinal fluid (CSF) detection of reduced $A \beta_{42}$, and/or high $A \beta_{40} / A \beta_{42}$ and high phosphorylated tau and total tau, or iii) neuronal injury or degeneration as shown by structural brain magnetic resonance imaging $(\mathrm{MRI})^{13}$. Tracking of brain pathology in longitudinal studies suggests that tau pathology may precede the accumulation of $A \beta$, but is undetectable as it is below current biomarker detection threshold levels, and is amplified catastrophically by independent A $\beta$ deposition ${ }^{14-16}$. ATN research framework based diagnosis of $A D$ is therefore limited by tau pathology detection, providing an impetus to develop specific and sensitive tau detection methods for earlier diagnosis.

Other factors to consider in the development of tau detection methods include the invasive nature of CSF sampling requiring lumbar puncture, and in the case of PET imaging, exposure to ionizing radiation, high cost, well documented side effects, irregular availability in primary care setting, and uneven geographical availability of PET scanners and isotopes ${ }^{17-21}$. The short half-life of PET agents also poses challenges for the detection of intracellular tau in the early stages of tau pathology formation ${ }^{22}$. Blood based markers are very promising, but only provide an indirect measure that cannot provide information on the localization of tau pathology in brain ${ }^{23-26}$. Methods to detect early tau pathology that avoid these pitfalls are therefore highly desirable.

The initiation of tau pathology is marked by abnormal phosphorylation of tau ${ }^{27,28}$. We hypothesize that hyperphosphorylative conditions in neurons, consistent with an altered balance of kinase-phosphatase activity resulting in elevated levels of hyperphosphorylated tau species, result in unique surface markers ${ }^{29-31}$. We envisioned a targeted contrast-enhanced MRI test identifying the earliest stages of tau pathology represented by 
cells in the process of abnormally phosphorylating tau. We used an iterative Cell-SELEX process to identify DNA thioaptamers that specifically bound such cells $s^{32,33}$. The use of phosphorothioate modified aptamers enhances stability under physiological conditions. High T1 relaxivity PEGylated liposomes bearing macrocyclic Gdchelates $^{34}$ were modified to bear the aptamers on their surface, thus enabling targeting of the particles to the surface of hyperphosphorylative cells for contrast-enhanced MRI. At the molecular level, we sought the binding targets of the aptamers, identifying vimentin, a normally intracellular protein that interestingly is specifically expressed on the surface of cells under hyperphosphorylative conditions, and representing a possible biomarker of pathological hyperphosphorylation found in $A D$.

\section{Results}

\section{Validation of cell surface changes in hyperphosphorylative conditions} in culture medium was used to model cell-surface changes under hyperphosphorylative conditions (Fig 1B). We used retinoic acid $(R A)$ to induce cell differentiation marked by temporal changes in morphology including the formation and lengthening of neurites and with a strong increase in levels of intracellular tau. Imbalance in the kinase and phosphatase activity leading to hyperphosphorylation, simulating early stages of tauopathies, was induced by the use of a cell permeable neurotoxin okadaic acid $(30 \mathrm{nM}, 24 \mathrm{~h})(\mathrm{OA})^{35}$, and confirmed by the increase in phosphorylated tau S396 (Supp. Fig. 1). In parallel experiments, a milder agent, excitotoxin quinolinic acid (QA) $1 \mu \mathrm{M}$ for $24 \mathrm{~h}$ was also used to induce hyperphosphosphorylation ${ }^{36}$.

A reverse phase protein array (RPPA) assay was conducted to test the hypothesis that hyperphosphorylation results in compositional changes reflected on the cell-surface. RPPA analysis demonstrated marked cell surface changes in hyperphosphorylative cells including over-expression of cell surface receptors. RPPA assessment with a panel of 221 proteins across different pathways and post-

81 translational modifications including phosphorylation examined SH SY5Y cell lysates under differentiated and hyperphosphorylative states. We identified 98 proteins and 36 phosphorylated proteins that showed significant change in their expression under different conditions (Fig 1C). Uniprot ${ }^{37}$ protein associations showed 44 cell- 


\section{Screening for aptamers that bind cells in hyperphosphorylative state}

Aptamer screening was performed using the cell-SELEX approach on differentiated SH-SY5Y cells in a hyperphosphorylative state (Fig. 2A). A total of 26 cell SELEX cycles were performed. To remove oligonucleotides that bound common cell surface molecules not specific to the hyperphosphorylative state, a negative selection was introduced at cycles 12 and 13 using differentiated, non hyperphosphorylative cells (i.e. without OA treatment). Anticipating that selected aptamers would be systemically delivered as nanoparticle imaging agents, and the primary toxicity driven by anomalous hepatocyte uptake, we conducted another round of negative selection at cycles 20 and 21 using a hepatocyte cell-line THLE-3 to remove oligonucleotides that exhibited enhanced uptake by hepatocytes.

\section{Tau1 and Tau3 aptamers specifically bind hyperphosphorylative cells}

Sequencing of all the selected pools was performed using the lon Torrent sequencing platform ${ }^{38}$ and revealed the evolution of families of DNA sequences, with enrichment particularly evident after 10 rounds of SELEX. Negative selection eliminated certain sequences that were not specific to the hyperphosphorylative state, or promoting hepatocyte uptake. However, the relative abundance of key sequences increased steadily throughout the whole process. The 23 most abundant sequences at round 26 were identified and their abundance throughout the SELEX process as calculated using AptaAligner ${ }^{39}$ is shown in Fig. $2 \mathrm{~B}$. The sequence Tau1 exemplifies this behavior representing a whole $59 \%$ of cycle 26 . A single base difference from this sequence, Tau3, is the second most represented sequence. The sequences present at the final round were grouped by hierarchical clustering and sequence homology using the multiple sequence alignment code MAFFT ${ }^{40}$ showing five distinct families (Fig 2E) also presented as a cladogram (using the Clustal Omega ${ }^{41}$ ) showing the common ancestry between these five aptamers families (Fig. 2F). Elevated levels of Tau1 binding to the membrane of hyperphosphorylative cells is demonstrated in Figure $2 \mathrm{C}$. The secondary structure of the aptamers Tau1 and Tau3 calculated using mfold ${ }^{42}$ is shown in Fig 2D. The apparent equilibrium dissociation constants $\left(\mathrm{Kd}_{\text {app }}\right)$ were measured by serial dilution of aptamer solutions with target hyperphosphorylated and non-target differentiated and undifferentiated SH-SY5Y cells. The affinity of these aptamers was also tested with another immortal neural progenitor stem cell line $\mathrm{ReN}-\mathrm{VM}^{43}$ in hyperphosphorylative and non-hyperphosphorylative conditions . The Kd app for Tau1 and Tau3 with hyperphosphorylated SH SY5Y cells is $0.167 \pm 0.015 \mathrm{nM}$ and $0.194 \pm 0.032$ nM; and for the ReN-VM cells $318.15 \pm 46.2 \mathrm{nM}$ and $234.24 \pm 38.6 \mathrm{nM}$ respectively (Supp. Fig 2). 


\section{TauX nanoparticles for magnetic resonance imaging}

For in vivo imaging of the hyperphosphorylative state in the brain of live mice, aptamer-targeted nanoparticles were fabricated for use as a molecular MRI contrast agent (TauX). TauX, was formulated as two versions, one using the Tau1 aptamer (TauT1) and another using the Tau3 aptamer (TauT3). Liposomal nanoparticles were synthesized using a lipid mixture that included lipidized Gd-DOTA for MR imaging, cholesterol for liposomal stability; additionally we also incorporated lipidized rhodamine for studying ex-vivo microscopic distribution of liposomal nanoparticles in brain tissues using fluorescence microscopy ${ }^{44,45}$. The TauX compositions also included DSPE-mPEG2000 to increase the in vivo circulation time ${ }^{34}$. Particles had a hydrodynamic diameter of $\sim 150 \mathrm{~nm}, \sim 86,000 \mathrm{Gd}$-chelates per liposomes and $\sim 500$ aptamers conjugated to the outer leaflet of each liposomal nanoparticle (Fig.3)

\section{In vivo molecular MRI using TauX for detection of hyperphosphorylative cells}

To test if TauX could detect the hyperphosphorylative state in vivo, MRI studies were performed in a P301S transgenic mouse model of AD-related tauopathy. Studies were performed in transgenic and agematched wild type mice at 2-3 months of age. At this young age, transgenic animals do not show frank tau pathology (i.e., neurofibrillary tangles), but practically all will develop tau pathology by around 8 months of age. Animals underwent baseline, pre-contrast MRI. Thereafter, animals were intravenously administered MRI contrast agent (TauT1, TauT3 or non-targeted control stealth liposomes that were not expected to provide signal enhancement as they did not have a targeting aptamer). Delayed post-contrast MRI was performed 4 days later. MR images were acquired using a T1-weighted spin-echo (T1w-SE) sequence and a T1-weighted fast spin echo inversion recovery (FSE-IR) sequence ${ }^{46}$. Transgenic mice administered TauT1 and TauT3 demonstrated signal enhancement in the cortex and the hippocampus regions of the brain (Fig. 4B). Wild-type mice (WT) administered TauT1 or TauT3 did not show signal enhancement in the brain. Similarly, transgenic mice administered nontargeted liposomal-Gd contrast agent did not show signal enhancement in cortex or hippocampus. These regions of interest were further analyzed quantitatively and signal-enhancement between the transgenic and wild-type mice were found to be statistically significant $(p<0.05)$ (Fig 4D). A baseline enhancement threshold of $\sim 6 \%(=2 X$ standard deviation of signal in baseline scans) was used as the classification threshold. Animals that showed signal enhancement above the threshold were identified as positives. Receiver operator characteristic (ROC) curves were generated with a six-point ordinal scale to assess sensitivity and specificity for detecting the 
genotype, using TauT1 and TauT3 contrast agents, and constructed over the entire tested group, including controls. The aptamer-targeted nanoparticle contrast agents, TauT1 and TauT3, showed overall AUC and accuracy of $\sim 0.95$. TauT3 demonstrated higher sensitivity than TauT1.

Post-mortem brain analysis was performed in 2-3 month old transgenic and wild-type mice. Immunofluorescence analysis using AT8 antibody staining revealed the presence of hyperphosphorylated tau species in transgenic mice but absent in wild type mice (Fig. 4F). A $100 \%$ concordance was observed between AT8 positivity and animal genotype. In summary, in vivo studies demonstrated that TauX enabled in vivo molecular MRI of the hyperphosphorylative state months before frank tau pathology i.e. the presence of neurofibrillary tangles, becomes evident in transgenic mice.

\section{Target identification of aptamers}

To characterize the binding target of the aptamers, we performed an aptamer-based pulldown assay, aptamer-based immunoprecipitation, followed by mass-spectrometry. We performed the assay for both Tau1 and Tau3, aptamers. A ranking of the abundance scores for identified proteins revealed keratin $6 \mathrm{a}$, Keratin $6 \mathrm{~b}$ and Vimentin as possible binding targets (Table 1). The surface expression of Keratin 6a, $6 \mathrm{~b}$ was similar on wild-type and transgenic tissue sections whereas the Vim expression was higher in the transgenic mice (Fig 5C). SHSY5Y cells under undifferentiated, and differentiated hyperphosphorylative conditions show increasing levels of vimentin (Fig.5B) further suggesting it is a potential target of aptamers Tau1 and Tau3.

\section{Discussion}

The ATN research framework suggests biomarkers to diagnose and classify AD. Under this framework CSF based detection of $A \beta$, tau (total, and phosphorylated) have been reported but only at the prodromal stage of disease, in patients with mild cognitive impairment ${ }^{47}$. Non-invasive neuroimaging tools, such as structural MRI to diagnose and monitor neurodegeneration ${ }^{48}$ show a definitive correlation with cognitive decline, visualizing atrophic regions that depict neuronal injury in late stage disease ${ }^{49}$. However, a reliable marker of early presymptomatic stage disease is yet to be identified.

While the role of $A \beta$ and tau in the development of $A D$ and the mechanism of transition from presymptomatic to symptomatic $A D$ are yet unclear, the time scale of the transition is generally accepted to be over a period of $10-20$ years $^{14}$. A $\beta$ deposits are considered the start of neurodegeneration but recent studies 
indicate that tau pathology ${ }^{50}$ shows a stronger correlation with disease progression suggesting that the limitation

170 of current tests is their inability to identify early stage pathological tau ${ }^{15}$. CSF presence of hyperphosphorylated

171 tau species $p-181$ and $p-217$ is associated with $A \beta$ deposition that precedes a positive tau PET ${ }^{51}$ but only has a

172 concordance of $50 \%-70 \%{ }^{52}$. Taken as a whole, the roles of $A \beta$ and tau deposition in disease progression and

173 the role of $A B$ in the spread of initial tau aggregates, strongly suggest that a biomarker of pathological tau at a

174 presymptomatic stage of the disease is likely to advance detection by several years, and constitutes the 175 motivation for this work.

Initial tau aggregation is thought to be triggered by an imbalance in cellular homeostasis caused by

177 dysregulated phosphorylation ${ }^{53,54}$. Several kinases can phosphorylate tau at multiple locations; at least 45 sites

178 have been observed experimentally ${ }^{55-57}$. Combined with reduced phosphatase activities in $A D$, the altered

179 kinase-phosphatase balance yields hyperphosphorylative conditions that cause abnormal hyperphosphorylation

180 of tau ${ }^{58}$. Disruption of the normal function of tau, modulating microtubule dynamics by lowering its binding

181 capabilities ${ }^{59}$ increases the level of cytosolic free tau leading to aggregation and fibrillization of tau that spreads

182 throughout the connected brain, seeding the pathology ${ }^{60,61}$. We hypothesized that this initial process of

183 hyperphosphorylation is associated with changes on the surface of hyperphosphorylative cells. We therefore sought to identify these surrogate markers of tau hyperphosphorylation that presage future tau pathology.

Using SH-SY5Y cells as a model of neuronal hyperphosphorylation, we used a reverse-phase protein array (RPPA) analysis to demonstrate elevated levels of surface molecules specific to the hyperphosphorylative

187 state (Fig 1C). Cell-SELEX capturing the differences between the surface of hyperphosphorylative cells and 188 normal cells allowed the selection of phosphorothioate modified short DNA aptamers that bound with high affinity 189 and specificity to hyperphosphorylative cells Fig 2 A. Having identified unique aptamers that bound such markers, 190 we developed MR molecular imaging contrast agents that recognize the surface of cells in hyperphosphorylative 191 state. We acknowledge that SH-SY5Y cells are not true neurons, they are a cell line originating in a 192 neuroblastoma, a tumor of embryological neural crest origin. However, they can be induced to differentiate to a 193 neuronal phenotype (as in the current work). While primary neuronal culture, or immortalized neuronal cells e.g. 194 ReN-VM may offer alternative models of neurons, we have functionally tested the aptamer hits from our SELEX 195 screen in a transgenic mouse model of tau deposition, and validated their performance, supporting our position 196 that the choice of cell model was adequate to identify suitable markers of tau hyperphosphorylation. 
PET is the leading modality for clinical molecular imaging, driven by its high contrast sensitivity, however,

198 it suffers from poor spatial resolution on the order of $5-10 \mathrm{~mm}$, high cost; limited access to radioactive tracers, and radiation exposure. Nanoparticle-enhanced MR imaging overcomes all these obstacles, but historically has not achieved high enough sensitivity. We have previously demonstrated liposomal nanoparticles exhibiting large numbers of Gd chelates in the external bilayer leaflet, with hyper-T1 relaxive properties resulting in contrast sensitivity that rival nuclear imaging ${ }^{45,62}$.

In P301S mice, the earliest reported histopathological studies are at age of 2.5 months $^{63,64}$, and report no tau pathology. "Tau seeding" the cell-cell transfer of pathogenic tau aggregates has been reported using brain homogenates at 1.5 month of age ${ }^{65}$. We therefore chose P301S mice at 2 months of age for our studies when, tau seeding should be taking place, but frank tau pathology should be absent. The mice were injected with TauX nanoparticles targeted either by the Tau1 aptamer or the Tau3 aptamer. When imaged by T1-weighted MRI sequences, designed to optimize signal from the Gd chelate induced T1 relaxation caused by the liposomal-Gd nanoparticles, signal enhancement was observed in the cortex and hippocampus regions of the brain. Hyperphosphorylative conditions were confirmed by post-mortem IF staining with AT8 antibody that recognizes 211 the S202 and T305 pTau species. Signal enhancement was not observed in non-transgenic mice, or in transgenic 212 mice injected with untargeted nanoparticles, supporting the specificity of Tau1-or Tau3-bearing nanoparticle 213 binding to target.

We have narrowed down the possible binding targets of the aptamers, and our data suggest that cell

215 surface vimentin is a likely target. We have confirmed the specific presence of cell surface vimentin on the surface of SH-SY5Y cells in a hyperphosphorylative state, and on P301S mouse brain sections. Vimentin is an

217 intermediate filament protein that undergoes constant assembly and/or remodeling and is usually associated with 218 mesenchymal cells ${ }^{66}$. The assembly state of filaments is linked to their phosphorylation state, phosphorylation 219 promotes disassembly ${ }^{67}$. Vimentin contains more than 35 phosphorylation sites targeted by multiple kinases and 220 phosphatases allowing it to adjust IF dynamics dependent on its environment ${ }^{68,69}$. Mechanical, chemical (toxins, 221 hypoxia), and microbial stresses upregulate vimentin and its phosphorylation that allows cells to adjust their 222 mechanical properties ${ }^{70,71}$. The balance of different oligomeric forms influence dynamic cell processes including 223 adhesion, migration, invasion including stress-induced signaling ${ }^{72}$. Vim IF's ( 10nm) distributed throughout the 224 cell by association with microtubules (tubulin, $24 \mathrm{~nm}$ ) regulating cell-migration, and microfilaments(actin, $7 \mathrm{~nm}$ ) 225 regulating cell-contractility, form the cytoskeletal network and provide mechanical support for the plasma 
membrane where it contacts other cells or the extracellular matrix ${ }^{73}$. Interestingly, during the biological process epithelial to mesenchymal transition wherein non-motile, polar epithelial cells transform to motile invasive nonpolar mesenchymal cells ${ }^{74}$, cells also undergo a cytoskeletal reorganization that includes changes in cellmembrane integrity, disassembly of junction proteins, increased stress-fiber formations, altered cell-surface protein expression. Changes in the localization of proteins is a hallmark of this pathologic process. Our observation that Vimentin is upregulated and translocated to the cell surface ${ }^{75}$ in the early stages of tau hyperphosphorylation suggests a possible role for EMT-related processes at the start of a slow progression towards AD pathology.

While positron emission tomography (PET) is the mainstay of molecular imaging, and exhibits remarkable sensitivity, there are several limitations posed by this methodology ${ }^{62,76}$. Access to PET imaging is limited, even in the relatively well-served US, and is skewed towards high density urban centers. PET costs are very high due to the need for same-day radiosynthesis, and rapid decay of the isotopes. Longer half-life isotopes cause higher radiation exposure. This tradeoff between half-life and radiation exposure greatly limit the reach of PET to a wider patient population. Current PET tau tracers recognize the tau $\beta$-sheets in the PHF and NFT present in tauopathies ${ }^{22}$. This conformation is not unique to tau and the in vivo specificity is circumspect limiting its interpretation. Off-target binding of Flortaucipir, an approved tau PET agent, has been reported since it binds the MAO-B enzyme in the brain $^{77,78}$. Further, the vast majority of pathological tau is actually intracellular, posing a significant barrier to PET tracers that must navigate to the site of tau pathology, bind the target, and have all unbound tracer molecules cleared from the brain before the radioactive signal decays. Our choice of MRI as the detection modality is based on hyper-T1 relaxive properties of nanoparticles with surface conjugated Gd chelates,

246 bringing detection sensitivity to the same range as nuclear imaging, and the MRI agent does not suffer from the 247 rapid signal decay of PET agents, allowing plenty of time for unbound tracer to clear from the brain before 248 imaging. Our choice of a cell surface surrogate marker of tau hyperphosphorylation avoids the need to bind an intracellular target. Finally, MRI imaging is already included in AD management and can be adjusted with agents such as TauX nanoparticles to constitute a highly sensitive and specific test for future tau pathology. 
254 of AD. Coupled with early amyloid detection, this test could enable early positive diagnosis using the NIA-AA

255 research framework.

256 Materials and Methods

257 Cell-lines - SH-SY5Y cells (ATCC, Manassas, VA, \#CRL-2266 ${ }^{\mathrm{TM}}$ ) were obtained from Dr. Jason Shohet's lab at 258 the Texas Children's Hospital, Houston, immortalized human hepatocytes (THLE-3) were purchased from 259 American Type Culture Collection (ATCC, Manassas, VA, \# CRL-11233 ${ }^{\mathrm{TM}}$ ); both were cultured according to the 260 ATCC instructions. ReN cell ${ }^{\text {TM }}$ VM (\#SCC008) cultured as per instruction using neural stem cell maintenance 261 medium (\#SCM005) and growth factors EGF (GF001) and bFGF(\#GF005) all from Millipore Sigma, Burlington, 262 MA.

263 Differentiation - SH-SY5Y cells were exposed to $30 \mu \mathrm{M}$ all-trans-Retionic acid (Sigma-Aldrich, St.Louis, MO, \# 264 R2625) in serum free cell medium for 10 days with medium change every alternate day. ReNcell VM were differentiated by the removal of growth factors from its culture medium for 10 days.

266 Hyperphosphorylation - was induced in SH-SY5Y cells by addition of $30 \mathrm{nM}$ okadaic acid (Sigma Aldrich, 267 St.Louis, MO, \# 459620) in growth medium with $30 \mu \mathrm{M}$ RA for 24 hours. ReNcell VM were hyperphosphorylated using 100nM Quinolinic Acid (SigmaAldrich, St.Louis, MO, \#P63204) in culture media for 24h.

Synthesis of primers and TA DNA library - All primers and Cy5, and amine labelled selected aptamers were purchased from Integrated DNA Technologies (IDT, Coralville, IA). The ssDNA library used in Cell-SELEX contained a central randomized sequence of 30 nucleotides flanked by PCR primer regions to enable the PCR

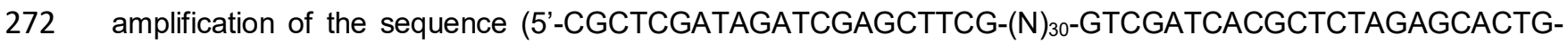
273 3'). The chemically synthesized DNA library was converted to a phosphorothioate modified library by PCR 274 amplification using, dATP (aS), resulting in the DNA sequences where the 3' phosphate of each residue is 275 substituted with monothiophosphate groups, as described previously in detail ${ }^{32,79}$. The reverse primer was 276 labeled with biotin to separate the sense strand from the antisense strand by streptavidin-coated sepharose 277 beads (PureBiotech, Middlesex, NJ, \# MSTR0510) for the next selection round. The concentration of the TA 278 library was determined with a NanoDrop ${ }^{\text {TM }} 2000$ by measuring the UV absorbance at $260 \mathrm{~nm}$.

279 Cell-SELEX - The procedures of Cell-SELEX were performed according to protocol previously with some 280 modification ${ }^{33,80}$. The initial ssDNA library of 150 pmole was dissolved in binding buffer with a total volume of 350 

SH-SY5Y cells at approximately $90 \%$ confluence in a 100-mm culture plate were washed twice with washing buffer and followed by incubating with the ssDNA library of 150 pmole for 2 hrs at $4^{\circ} \mathrm{C}$. Following the incubation,

284 for positive selections, the supernatant was discarded, and cells were washed three times with washing buffer to remove any unbound sequences. Then cells were scraped off and transferred to nuclease-free water, following another three times nuclease-free water washes. Cells in nuclease-free water was centrifuged at $300 \times \mathrm{g}$ for 5 min. QIAamp DNA Mini and Blood Mini kit (Qiagen, Germantown, MD, \# 51104) was introduced to elute cell membrane fraction. The cell membrane fraction was PCR-amplified to monitor the presence of cell binding efficacy at each cycle. For negative selections, the supernatant was simply pipetted out of the flask and processed for the next cycle of selection. The desired compartment were amplified by PCR, and used to prepare the TA for the next round of selection. Two different negative selections were involved. One was differentiated treatment only SH-SY5Y cells at cycles \#12 and \#13. Another was hepatocyte THLE-3 cells at cycles \#20 and \#21. A total of 26 cycles of Cell-SELEX were conducted, including two different types of negative selections mentioned above.

Next-Generation Sequencing (NGS) - At the studied cycles, the membrane fractions were isolated and the recovered TA sequences were amplified by PCR. Equimolar quantities of the recovered TA sequences over the range were pooled together and sequenced by Next-Gen DNA sequencing using lon318 chip (ThermoFisher, Waltham, MA). A four base sequence was introduced during PCR amplification to serve as unique "barcode" to distinguish between the studied cycles. Sequencing results were analyzed by the Aptalinger ${ }^{39}$ that uses the markov model probability theory to find the optimal alignment of the sequences.

Aptamer binding studies - were conducted with undifferentiated, differentiated and hyperphosphorylated SH SY5Y and ReN cell VM grown in 96-wells seeded at 10000 per well. The apparent dissociation constants (Kds) were measured by the equation $Y=B \max X /(K d+X)$, with GraphPad Prism 9, San Diego, CA, with a saturation binding experiment; cells were incubated with varying concentrations of Cy5-labeled aptamer in a $100 \mu$ volume of binding buffer containing cells, incubated for 30minutes, washed twice and resuspended in $100 \mu$ buffer and analyzed by Molecular probes microplate reader equipped with the appropriate excitation and emission filters.

307 All data points were collected in triplicate. 
and air dried prior to seeding 20,000 SH-SY5Y cells per well. Aptamer staining at $100 \mathrm{nM}$ was performed with live cells for $2 \mathrm{~h}$ at $4^{\circ} \mathrm{C}$ in binding buffer and washed twice with washing buffer. Cells were then fixed by incubation

312 for $15 \mathrm{~min}$ in $4 \%$ formaldehyde in PBS at room temperature. Non-specific binding was blocked with blocking 313 buffer (G-Biosciences, St. Louis, MO, \# 786195) for 1 hour and overnight incubation at $4^{\circ} \mathrm{C}$ with the rabbit pTau 314 primary antibody (1:100) (Santa Cruz Biotechnology, \#: sc-101815) was followed by washing with PBS, and 1h 315 incubation with goat anti-rabbit IgG secondary antibody, Alexa Fluor 488 (Invitrogen, Carlsbad, CA, \# A-11008) for 1 hour at room temperature. Cytoskeletal actin filaments were stained with Alexa Fluor 594 Phalloidin

317 (Invitrogen, \# A12381). The cells were covered with VECTASHIELD hardset mounting medium with DAPI (Vector 318 Laboratories, Burlingame, CA, \# H-1500) for 5 min at room temperature. Images were visualized under Olympus 319 Fluoview FV1000 confocal microscopy.

TauX nanoparticle synthesis - L-a-phosphatidylcholine, hydrogenated (Hydro Soy PC; HSPC) and Cholesterol were purchased from Lipoid Inc., Newark NJ, USA. 1,2-distearoyl-sn-glycero-3-phosphoethanolamine-N322 [methoxy(polyethylene glycol)-2000] (DSPE-mPEG2000) was purchased from Corden Pharma, Liestahl, 323 Switzerland. DSPE-PEG3400-COOH and Gd-DOTA-DSPE were synthesized in house, lis-rhodamine-DHPE 324 from ThermoFisher Scientific. HSPC, Cholesterol, DSPE-PEG3400-COOH, DSPE-mPEG2000, Gd-DOTADSPE, lis-rhodamine-DHPE at molar proportions 31.4:40:0.5:3:25:0.1 were dissolved in ethanol to achieve a total concentration of $100 \mathrm{mM}$. For the non-targeted control stealth liposomes, carboxy terminated PEG was not included in the lipid mixture. The ethanolic solution of lipids was hydrated with $150 \mathrm{mM}$ saline solution at $65^{\circ} \mathrm{C}$ for 30 minutes, allowing multilamellar liposomes to form. The mixture was then extruded in a $10 \mathrm{ml}$ Lipex extruder (Northern Lipids Inc., Burnaby, Canada) using a $400 \mathrm{~nm}$ polycarbonate track-etch polycarbonate filter (3 passes) followed by a $200 \mathrm{~nm}$ ( 3 passes) and finally 100nM filters. The suspension was then diafiltered using a MicroKros cross-flow diafiltration cartridge (500 kDa cutoff) from Repligen, Rancho Dominguez, CA, exchanging the external buffer for phosphate buffered saline (PBS, $\mathrm{pH}$ 7.2) for 15 volume exchanges. To form the aptamer conjugated liposomes, liposomes with lipid-PEG-COOH were reacted with amine terminated aptamers using carbodiimide chemistry. The carboxyl groups on the liposomes were activated with $5 \mathrm{mM}$ EDC and $10 \mathrm{mM}$ sulfo-NHS at pH 6 for 5-10 minutes. The activated liposomes were then immediately reacted with the amine terminated aptamers and the $\mathrm{pH}$ was raised to $\sim 7.3-7.6$ by titrating $\mu \mathrm{l}$ amounts of $5 \mathrm{~N} \mathrm{NaOH}$. The final concentration of aptamers used in reaction is $\sim 140 \mu \mathrm{M}$. The reaction was mixed at room temperature for $1 \mathrm{hr}$ following which the reaction was carried out at $4 \mathrm{deg}{ }^{\circ} \mathrm{C}$ overnight. The liposomes were then dialyzed against PBS to remove unconjugated 
aptamers using a $300 \mathrm{kDa}$ dialysis membrane. The dialysate (external phase) was concentrated using $10 \mathrm{kD}$ centrifugal separator and washed with PBS to remove residual EDC/s-NHS. The concentrated dialysate was analysed by NanoDrop Spectrophotometer (ThermoFisher Sci., Waltham, MA, USA) to determine unconjugated aptamer fraction, and estimate aptamer density per nanoparticle in TauX formulations. Inductively coupled plasma atomic emission spectroscopy (ICP-AES) was used to measure Gd and phosphorus concentrations of TauX formulations. The hydrodynamic diameter of liposomal nanoparticles in TauX formulations was determined using a dynamic light scattering instrument.

Mice - All the procedures were performed with approval from Institutional Animal Care and Use Committee (IACUC) of Baylor College of Medicine. Mice were kept under a $12 \mathrm{~h} \mathrm{light/dark} \mathrm{cycle,} \mathrm{with} \mathrm{food} \mathrm{and} \mathrm{water} \mathrm{available}$ ad libitum. PS19 mice from Jackson Laboratories (Bar Harbor, ME) B6; C3-Tg (Prnp-MAPTP301S) PS19Vle/J Stock No: 008169 were used and experiments were conducted at the $2 \mathrm{~m}$ of age. The transgenic (TG) mice develop neurofibrillary tangles by 5 months of age ${ }^{64}$. Age-matched non-transgenic wild type (WT) mice were used as controls.

Magnetic Resonance Imaging (MRI) - MRI was performed on a 1T permanent magnet scanner (M7, Aspect Imaging, Shoham, Israel). Mice underwent pre-contrast baseline scans. Thereafter, mice were intravenously administered one of three nanoparticle MR contrast agents (TauT1, TauT3 or non-targeted control liposomes) via tail vein at a dose of $0.20 \mathrm{mmol} \mathrm{Gd} / \mathrm{kg}$ of body weight. Delayed post-contrast MRI was performed 4 days after contrast agent injections. Pre-contrast and delayed post-contrast MR images were acquired using a T1-weighted spin echo (T1W-SE) sequence and a fast spin echo inversion recovery (FSE-IR) sequence with the following parameters: $\underline{\text { SE parameters: }} \mathrm{TR}=600 \mathrm{~ms}, \mathrm{TE}=11.5 \mathrm{~ms}$, slice thickness $=1.2 \mathrm{~mm}$, matrix $=192 \times 192, \mathrm{FOV}=30$ $\mathrm{mm}$, slices $=16, \mathrm{NEX}=4 ;$ FSE-IR parameters: $\mathrm{TR}=13500 \mathrm{~ms}, \mathrm{TE}=80 \mathrm{~ms}, \mathrm{TI}=2000 \mathrm{~ms}$, slice thickness $=2.4$ $\mathrm{mm}$, matrix $=192 \times 192, \mathrm{FOV}=30 \mathrm{~mm}$, slices $=6, \mathrm{NEX}=6$. Coil calibration, RF calibration, and shimming were

361 performed at the beginning of study for each subject. The pre-contrast scans provide a baseline for calculation 362 of signal enhancement from resulting post-contrast scans ${ }^{46}$. Two-standard deviations above the mean variation 363 within WT control animals was used as the cutoff signal intensity for identifying tau positive animals. Six 364 transgenic (TG) mice and six wild type mice (WT) were used for testing of each nanoparticle contrast agent 365 formulation. Receiver operating characteristic (ROC) curves were generated on a six point ordinal scale by 366 plotting the true positive fraction (TPF) against the false positive fraction (FPF) based on imaging-based identification of Tau-positive animals using the cutoff signal intensity and then comparing against histological 
confirmation of Tau pathology as a gold standard. A fitted curve was then generated against the empirical points plotted on the graphs. Qualitative and quantitative analysis of MRI images was performed in OsiriX (version 5.8.5, 64-bit, Pixmeo SARL, Geneva, Switzerland) and MATLAB (version 2015a, MathWorks, Natick, MA). saline followed by $4 \%$ paraformaldehyde for 15 mins. The brains were then immersion-fixed in $4 \%$ formaldehyde for $48 \mathrm{~h}$ at $4^{\circ} \mathrm{C}$, transferred to $30 \%$ sucrose for cryoprotection and embedded in OCT. Phenotypic confirmation for the presence of phosphorylated tau and vimentin was done on $25 \mu \mathrm{m}$ thick brain sections. Antigen retrieval in $\mathrm{pH}=8.5$ citrate buffer was executed in a $1200 \mathrm{~W}$ GE microwave for 15 mins. After 15 minutes of cooling $25 \mu \mathrm{L}$ of 1:50 dilution of primary p-tau antibody namely either AT8,AT100 or AT180 that recognize different p-tau species were incubated in a tray (RPI, Mt. Prospect, IL \#248270) designed for microwave enhanced immunostaining procedures for $3 \mathrm{~min}$ at power level 3. After a 2 min cooling, sections were washed with PBS and incubated for 3min with a 1:100 dilution of appropriate secondary antibody. DAPI staining proceeded after 2 mins of cooling and a PBS washing. ProGold Antifade (Invitrogen, Carlsbad, CA, \# P36030) was used to mount slides which were visualized on Olympus Fluoview LV100. Scanning of whole sections was also conducted using a Biotek Cytation 5 slide scanning microscope. List of Antibodies - AT8 (\#MN1020), Vimentin SP20 (\#MA516409) both Thermo Fisher Scientific, Waltham, MA, Vimentin D21H3 (Cell Signaling Technology, Beverly, MA, \#5741T,), Cell-surface vimentin (Abnova, Taipei City, Taiwan, \#H00007431-M08J).

Target Identification - The protein targets of Tau-1, Tau-3, Tau-4 and Tau-5 were identified by affinity-pull down using the selected aptamers as the capturing reagent followed by mass-spectroscopy. A scrambled DNA sequence, R2, was used as a control. The hyperphosphorylated SH-SY5Y cells, at 90-95\% confluence were washed with cold PBS buffer and incubated with biotinylated selected aptamers with $25 \mathrm{mmol} / \mathrm{l}$ each at $4^{\circ} \mathrm{C}$ in PBS, respectively. After 2 hours of gentle agitation, SH-SY5Y cells were cross-linked with $1 \%$ formaldehyde for 10 minutes at room temperature. The formaldehyde cross-linking was quenched with glycine. Cells were scraped 391 from the plate, washed and lysed with lysing buffer (Thermofisher Scientific, \# 87787) and treated with protease 392 inhibitor mixture. The lysates were freeze-thawed for 30 minutes on ice and cleared by centrifuging at $10,000 \times g$ 393 for 2 minutes at $4^{\circ} \mathrm{C}$. To pull down the cross-linked proteins, equal amounts of cell lysate were incubated with 394 prewashed streptavidin magnetic beads for 1 hour at room temperature with continuous rotation. Protein 395 digestions were performed on the beads to isolate targeted proteins and processed for mass spectrometric analysis. Each sample was analyzed in triplicates. The raw data files were processed to generate a Mascot 
397 Generic Format with Mascot Distiller and searched against the SwissProt_2012_01 (Human) database using the

398 licensed Mascot search engine v2.3.02 (Matrix Science, Boston, MA) run on an in-house server.

\section{References}

400 1. Goedert, M., Wischik, C. M., Crowther, R. A., Walker, J. E. \& Klug, A. Cloning and sequencing of the 401 cDNA encoding a core protein of the paired helical filament of Alzheimer disease: identification as the 402 microtubule-associated protein tau. Proc. Natl. Acad. Sci. U. S. A. 85, 4051-4055 (1988).

403 2. Goedert, M., Spillantini, M. G., Jakes, R., Rutherford, D. \& Crowther, R. A. Multiple isoforms of human 404 microtubule-associated protein tau: sequences and localization in neurofibrillary tangles of Alzheimer's 405 disease. Neuron 3, 519-526 (1989).

406 3. lqbal, K., Liu, F. \& Gong, C. Tau and neurodegenerative disease: the story so far. Nat. Rev. Neurol. 12, 15-27 (2016).

408

4. Guo, T., Noble, W. \& Hanger, D. Roles of tau protein in health and disease. Acta Neuropathol. 133, 665-704 (2017).

410 5. Wang, Y. \& Mandelkow, E. Tau in physiology and pathology. Nat. Rev. Neurosci. 17, 22 (2016).

411 6. Chong, F. P., Ng, K. Y., Koh, R. Y. \& Chye, S. M. Tau Proteins and Tauopathies in Alzheimer's

412 Disease. Cell. Mol. Neurobiol. 38, 965-980 (2018).

413 7. Karikari, T. K. et al. Distinct conformations, aggregation and cellular internalization of different tau strains. Front. Cell. Neurosci. 13, 1-16 (2019).

415 8. d'Errico, P. \& Meyer-Luehmann, M. Mechanisms of Pathogenic Tau and A $\beta$ Protein Spreading in Alzheimer's Disease. Front. Aging Neurosci. 12, 1-10 (2020).

417 9. Brunello, C. A., Merezhko, M., Uronen, R. L. \& Huttunen, H. J. Mechanisms of secretion and spreading of pathological tau protein. Cell. Mol. Life Sci. 77, 1721-1744 (2020).

419 10. Vogel, J. W. et al. Spread of pathological tau proteins through communicating neurons in human Alzheimer's disease. Nat. Commun. 11, 2612 (2020).

421 11. Gibbons, G. S., Lee, V. M. Y. \& Trojanowski, J. Q. Mechanisms of Cell-to-Cell Transmission of 
423 12. Jack Jr, C. R. et al. NIA-AA research framework: toward a biological definition of Alzheimer's disease. Alzheimer's Dement. 14, 535-562 (2018).

425 13. Jack, C. R. et al. A/T/N: an unbiased descriptive classification scheme for Alzheimer disease biomarkers. Neurology 87, 539-547 (2016).

14. Selkoe, D. J. \& Hardy, J. The amyloid hypothesis of Alzheimer's disease at 25 years. EMBO Mol. Med. 8, 595-608 (2016).

15. Mattsson-Carlgren, N. et al. A $\beta$ deposition is associated with increases in soluble and phosphorylated tau that precede a positive Tau PET in Alzheimer's disease. Sci. Adv. 6, (2020).

16. Leuzy, A. et al. Longitudinal tau and metabolic PET imaging in relation to novel CSF tau measures in Alzheimer's disease. Eur. J. Nucl. Med. Mol. Imaging 46, 1152-1163 (2019).

17. Cummings, J. L. Biomarkers in Alzheimer's disease drug development. Alzheimer's Dement. 7, e13e44 (2011).

18. Smoler, M., Coceano, G., Testa, I., Bruno, L. \& Levi, V. Apparent stiffness of vimentin intermediate Cell Res. 1867, 118726 (2020).

19. Peskind, E., Nordberg, A., Darreh-Shori, T. \& Soininen, H. Safety of lumbar puncture procedures in patients with Alzheimer's disease. Curr. Alzheimer Res. 6, 290-292 (2009).

20. Wittenberg, R., Knapp, M., Karagiannidou, M., Dickson, J. \& Schott, J. M. Economic impacts of introducing diagnostics for mild cognitive impairment Alzheimer's disease patients. Alzheimer's Dement. Transl. Res. Clin. Interv. 5, 382-387 (2019).

443 21. Ehman, E. C. et al. PET/MRI: where might it replace PET/CT? J. Magn. Reson. Imaging 46, 1247-1262 (2017).

445 22. Leuzy, A. et al. Tau PET imaging in neurodegenerative tauopathies-still a challenge. Mol. Psychiatry 
447 23. Jack Jr, C. R. et al. Tracking pathophysiological processes in Alzheimer's disease: an updated hypothetical model of dynamic biomarkers. Lancet Neurol. 12, 207-216 (2013).

24. Terry, R. D. et al. Physical basis of cognitive alterations in Alzheimer's disease: synapse loss is the major correlate of cognitive impairment. Ann. Neurol. Off. J. Am. Neurol. Assoc. Child Neurol. Soc. 30,

25. Hyman, B. T. et al. National Institute on Aging-Alzheimer's Association guidelines for the neuropathologic assessment of Alzheimer's disease. Alzheimer's Dement. 8, 1-13 (2012).

26. Montine, T. J. et al. National Institute on Aging-Alzheimer's Association guidelines for the neuropathologic assessment of Alzheimer's disease: a practical approach. Acta Neuropathol. 123, 1-11

27. Wang, J.-Z., Xia, Y.-Y., Grundke-lqbal, I. \& Iqbal, K. Abnormal hyperphosphorylation of tau: sites, regulation, and molecular mechanism of neurofibrillary degeneration. J. Alzheimer's Dis. 33, S123S139 (2013).

28. Grundke-lqbal, I. et al. Abnormal phosphorylation of the microtubule-associated protein tau (tau) in Alzheimer cytoskeletal pathology. Proc. Natl. Acad. Sci. 83, 4913-4917 (1986).

29. Delobel, P. et al. Analysis of tau phosphorylation and truncation in a mouse model of human tauopathy. Am. J. Pathol. 172, 123-131 (2008).

30. Lund, E. T., McKenna, R., Evans, D. B., Sharma, S. K. \& Mathews, W. R. Characterization of the in

31. Liu, F., Grundke-lqbal, I., Iqbal, K. \& Gong, C. Contributions of protein phosphatases PP1, PP2A, PP2B and PP5 to the regulation of tau phosphorylation. Eur. J. Neurosci. 22, 1942-1950 (2005).

469 32. Mu, Q. et al. Conjugate-SELEX: A High-throughput Screening of Thioaptamer-liposomal Nanoparticle Conjugates for Targeted Intracellular Delivery of Anticancer Drugs. Mol. Ther. - Nucleic Acids 5, e382 (2016).

472 33. Parekh, P., Tang, Z., Turner, P. C., Moyer, R. W. \& Tan, W. Aptamers Recognizing Glycosylated 
Hemagglutinin Expressed on the Surface of Vaccinia Virus-Infected Cells. Anal. Chem. 82, 8642-8649 (2010).

34. Ayyagari, A. L. et al. Long-circulating liposomal contrast agents for magnetic resonance imaging. Magn. Reson. Med. An Off. J. Int. Soc. Magn. Reson. Med. 55, 1023-1029 (2006).

35. Kamat, P. K., Rai, S., Swarnkar, S., Shukla, R. \& Nath, C. Molecular and Cellular Mechanism of Okadaic Acid (OKA)-Induced Neurotoxicity: A Novel Tool for Alzheimer's Disease Therapeutic Application. Mol. Neurobiol. 50, 852-865 (2014).

36. Gulaj, E., Pawlak, K., Bien, B. \& Pawlak, D. Kynurenine and its metabolites in Alzheimer's disease patients. Adv. Med. Sci. 55, 204-211 (2010).

37. The UniProt Consortium. UniProt: the universal protein knowledgebase in 2021. Nucleic Acids Res. 49, D480-D489 (2021).

38. Rothberg, J. M. et al. An integrated semiconductor device enabling non-optical genome sequencing. Nature 475, 348-352 (2011).

39. Lu, E., Elizondo-Riojas, M.-A., Chang, J. T. \& Volk, D. E. Aptaligner: automated software for aligning pseudorandom DNA X-aptamers from next-generation sequencing data. Biochemistry 53, 3523-3525 (2014).

40. Katoh, K., Rozewicki, J. \& Yamada, K. D. MAFFT online service: multiple sequence alignment, interactive sequence choice and visualization. Brief. Bioinform. 20, 1160-1166 (2019).

41. Sievers, F. et al. Fast, scalable generation of high-quality protein multiple sequence alignments using Clustal Omega. Mol. Syst. Biol. 7, 539 (2011).

42. Zuker, M. Mfold web server for nucleic acid folding and hybridization prediction. Nucleic Acids Res. 31, $3406-3415$ (2003).

43. Donato, R. et al. Differential development of neuronal physiological responsiveness in two human neural stem cell lines. BMC Neurosci. 8, 1-11 (2007).

44. Ghaghada, K., Hawley, C., Kawaji, K., Annapragada, A. \& Mukundan Jr, S. T1 relaxivity of coreencapsulated gadolinium liposomal contrast agents—effect of liposome size and internal gadolinium 
concentration. Acad. Radiol. 15, 1259-1263 (2008).

500

501

502

503

504

505

506

507

508

509

510

511

512

45. Ghaghada, K. B. et al. New Dual Mode Gadolinium Nanoparticle Contrast Agent for Magnetic Resonance Imaging. PLoS One 4, e7628 (2009).

46. Badachhape, A. A. et al. Pre-clinical dose-ranging efficacy, pharmacokinetics, tissue biodistribution, and toxicity of a targeted contrast agent for MRI of amyloid deposition in Alzheimer's disease. Sci. Rep. 10, $16185(2020)$.

47. Mattsson, N., Lönneborg, A., Boccardi, M., Blennow, K. \& Hansson, O. Clinical validity of cerebrospinal fluid $A \beta 42$, tau, and phospho-tau as biomarkers for Alzheimer's disease in the context of a structured 5phase development framework. Neurobiol. Aging 52, 196-213 (2017).

48. Coch, R. A. \& Leube, R. E. Intermediate filaments and polarization in the intestinal epithelium. Cells 5, $32(2016)$.

49. Cuingnet, R. et al. Automatic classification of patients with Alzheimer's disease from structural MRI: a comparison of ten methods using the ADNI database. Neuroimage 56, 766-781 (2011).

50. Johansson, M. et al. Mild behavioral impairment and its relation to tau pathology in preclinical Alzheimer's disease. Transl. Psychiatry 11, 1-8 (2021).

51. Suárez-Calvet, M. et al. Novel tau biomarkers phosphorylated at T181, T217 or T231 rise in the initial stages of the preclinical Alzheimer's continuum when only subtle changes in A $\beta$ pathology are detected. EMBO Mol. Med. 12, e12921 (2020).

52. Mattsson, N. et al. 18 F-AV-1451 and CSF T-tau and P-tau as biomarkers in Alzheimer's disease . EMBO Mol. Med. 9, 1212-1223 (2017).

53. Chung, S.-H. Aberrant phosphorylation in the pathogenesis of Alzheimer's disease. BMB Rep. 42, 467474 (2009).

54. Oliveira, J., Costa, M., de Almeida, M. S. C., da Cruz e Silva, O. A. B. \& Henriques, A. G. Protein phosphorylation is a key mechanism in Alzheimer's disease. J. Alzheimer's Dis. 58, 953-978 (2017).

55. Augustinack, J. C., Schneider, A., Mandelkow, E.-M. \& Hyman, B. T. Specific tau phosphorylation sites correlate with severity of neuronal cytopathology in Alzheimer's disease. Acta Neuropathol. 103, 26-35 
56. Wang, Y. \& Mandelkow, E. Tau in physiology and pathology. Nat. Rev. Neurosci. 17, 22-35 (2016).

527 57. Wang, J., Grundke-lqbal, I. \& Iqbal, K. Kinases and phosphatases and tau sites involved in Alzheimer neurofibrillary degeneration. Eur. J. Neurosci. 25, 59-68 (2007).

58. Sontag, J.-M. \& Sontag, E. Protein phosphatase 2A dysfunction in Alzheimer's disease. Front. Mol. Neurosci. 7, 16 (2014).

59. Sontag, J. M., Nunbhakdi-Craig, V., White, C. L., Halpain, S. \& Sontag, E. The protein phosphatase PP2A/Ba binds to the microtubule-associated proteins Tau and MAP2 at a motif also recognized by the kinase Fyn: Implications for tauopathies. J. Biol. Chem. 287, 14984-14993 (2012).

60. Franzmeier, N. et al. Functional brain architecture is associated with the rate of tau accumulation in Alzheimer's disease. Nat. Commun. 11, 1-17 (2020).

61. DeVos, S. L. et al. Synaptic tau seeding precedes tau pathology in human Alzheimer's disease brain. Front. Neurosci. 12, 267 (2018).

62. Dustin, D., Hall, B. M., Annapragada, A. \& Pautler, R. G. Neuroimaging in Alzheimer's disease: preclinical challenges toward clinical efficacy. Transl. Res. 175, 37-53 (2016).

63. Takeuchi, H. et al. P301S mutant human tau transgenic mice manifest early symptoms of human tauopathies with dementia and altered sensorimotor gating. PLoS One 6, e21050 (2011).

542 64. Yoshiyama, Y. et al. Synapse loss and microglial activation precede tangles in a P301S tauopathy mouse model. Neuron 53, 337-351 (2007).

544 65. Holmes, B. B. et al. Proteopathic tau seeding predicts tauopathy in vivo. Proc. Natl. Acad. Sci. 111, E4376 LP-E4385 (2014).

546 66. Eriksson, J. E. et al. Introducing intermediate filaments: from discovery to disease. J. Clin. Invest. 119, 1763-1771 (2009).

548 67. Sihag, R. K., Inagaki, M., Yamaguchi, T., Shea, T. B. \& Pant, H. C. Role of phosphorylation on the 549 structural dynamics and function of types III and IV intermediate filaments. Exp. Cell Res. 313, 2098- 
2109 (2007).

68. Snider, N. T. \& Omary, M. B. Post-translational modifications of intermediate filament proteins: mechanisms and functions. Nat. Rev. Mol. Cell Biol. 15, 163-177 (2014).

69. Battaglia, R. A., Delic, S., Herrmann, H. \& Snider, N. T. Vimentin on the move: New developments in cell migration [version 1; referees: 2 approved]. F1000Research 7, 1-10 (2018).

70. Pattabiraman, S. et al. Vimentin protects differentiating stem cells from stress. Sci. Rep. 10, 1-15 (2020).

71. Etienne-Manneville, S. Cytoplasmic Intermediate Filaments in Cell Biology. Annu. Rev. Cell Dev. Biol. 34, 1-28 (2018).

72. Ivaska, J., Pallari, H.-M., Nevo, J. \& Eriksson, J. E. Novel functions of vimentin in cell adhesion, migration, and signaling. Exp. Cell Res. 313, 2050-2062 (2007).

73. Jones, J. C. R. et al. Intermediate filaments and the plasma membrane. Cold Spring Harb. Perspect. Biol. 9, (2017).

74. Kalluri, R. EMT: when epithelial cells decide to become mesenchymal-like cells. J. Clin. Invest. 119, 1417-1419 (2009).

75. Ivaska, J. Vimentin: Central hub in EMT induction? Small GTPases 2, 1436-1448 (2011).

76. Hameed, S. et al. Role of Fluid Biomarkers and PET Imaging in Early Diagnosis and its Clinical Implication in the Management of Alzheimer's Disease. J. Alzheimer's Dis. Reports 4, 21-37 (2020).

77. Lemoine, L. et al. Comparative binding properties of the tau PET tracers THK5117, THK5351, PBB3, and T807 in postmortem Alzheimer brains. Alzheimers. Res. Ther. 9, 1-13 (2017).

78. Baker, S. L., Harrison, T. M., Maass, A., La Joie, R. \& Jagust, W. J. Effect of off-target binding on 18FFlortaucipir variability in healthy controls across the life span. J. Nucl. Med. 60, 1444-1451 (2019).

79. Volk, D. E. \& Lokesh, G. L. R. Development of phosphorothioate DNA and DNA thioaptamers. Biomedicines 5, 41 (2017).

80. $\mathrm{Mu}, \mathrm{Q}$. et al. Conjugate-SELEX: A high-throughput screening of thioaptamer-liposomal nanoparticle 
bioRxiv preprint doi: https://doi.org/10.1101/2021.05.14.444233; this version posted May 17, 2021. The copyright holder for this preprint (which

was not certified by peer review) is the author/funder, who has granted bioRxiv a license to display the preprint in perpetuity. It is made available under aCC-BY-NC-ND 4.0 International license.

575 conjugates for targeted intracellular delivery of anticancer drugs. Mol. Ther. Acids 5, e382 (2016).

576 Acknowledgments: We acknowledge the work of Dr. Igor Stupin (now deceased) in the handling, treatment,

577 and necropsy of all animals. Funding: This work was supported by Alzeca Biosciences, Inc. grants SRA 1, 2

578 and 3 to A.A. 


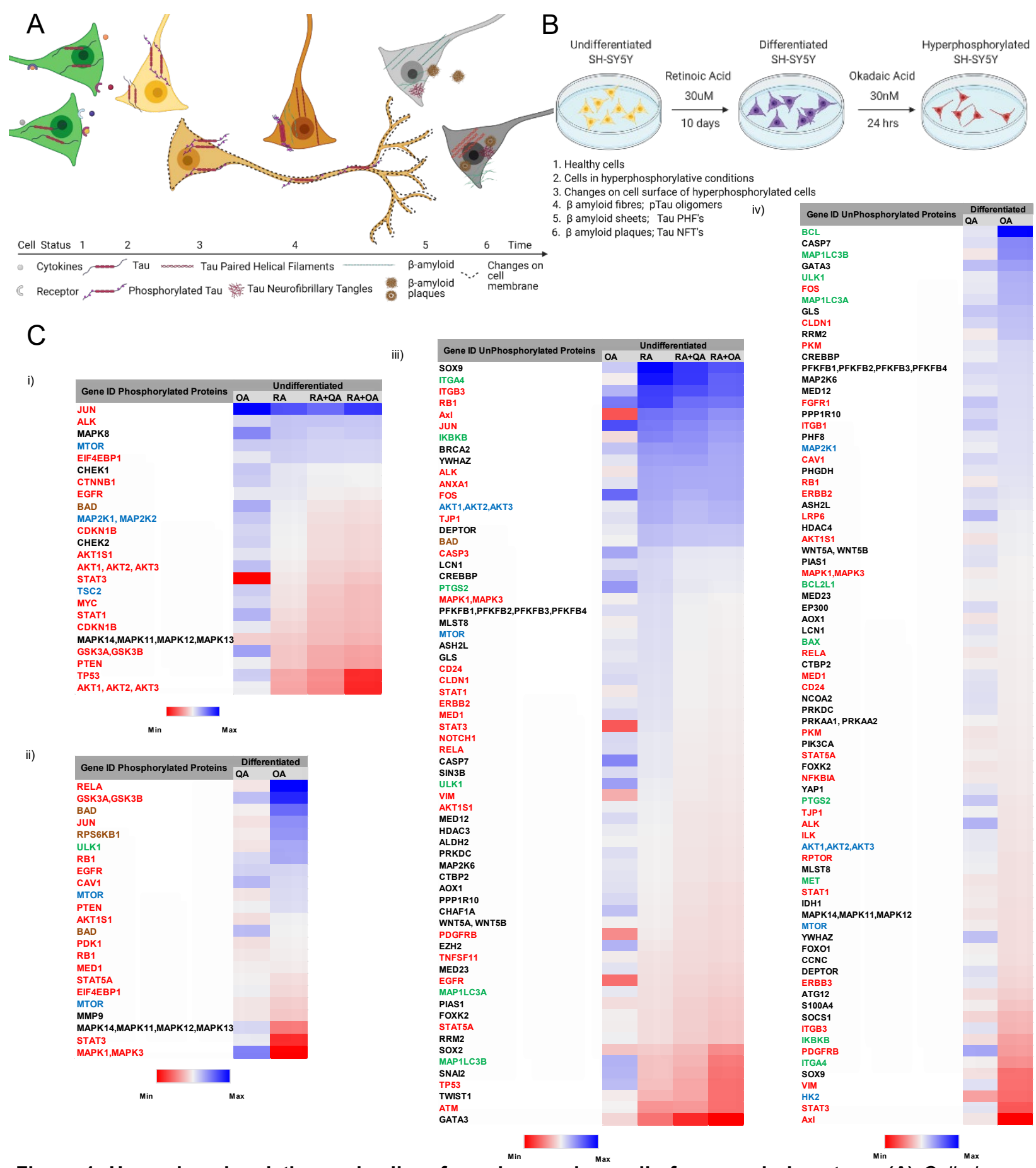

Figure 1: Hyperphosphorylation and cell surface changes in a cell of neuronal phenotype. (A) Cell changes during hyperphosphorylation: Tau hyperphosphorylation is a precursor to tau fibrillation. We hypothesize that cell surface changes occur in parallel with tau hyperphosphorylation. These changes may constitute a marker for early detection of AD. (B) In vitro model of hyperphosphorylation in a cell of neuronal phenotype: Differentiated SH-SY5Y cells are used to model hyperphosphorylation. Hyperphosphorylation can be induced by either okadaic acid (OA) or quinolinic acid (QA) (C) Protein analysis at different stages of hyperphosphorylation: Reverse phase protein array analysis of undifferentiated, retinoic acid (RA) differentiated and hyperphosphorylative cells (by Okadaic acid OA or Quinolinic acid QA). Heat map depicts changes in total protein expression. Proteins expressed on cell-membrane are depicted in red, peripheral -membrane proteins in blue and single-pass and multipass membrane proteins in green. Changes due to phosphorylation treatments with OA and QA on i) undifferentiated and ii) differentiated SH-SY5Y cells. Changes in un-phosphorylated proteins on iii) undifferentiated and iv) differentiated SH-SY5Y cells. 
A and
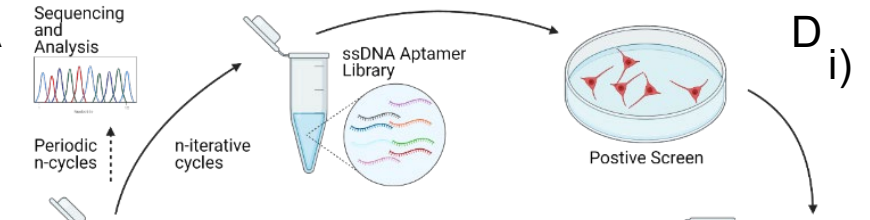

i)

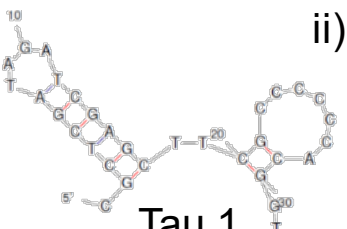

ii) ${ }_{A}^{10}$ G-A
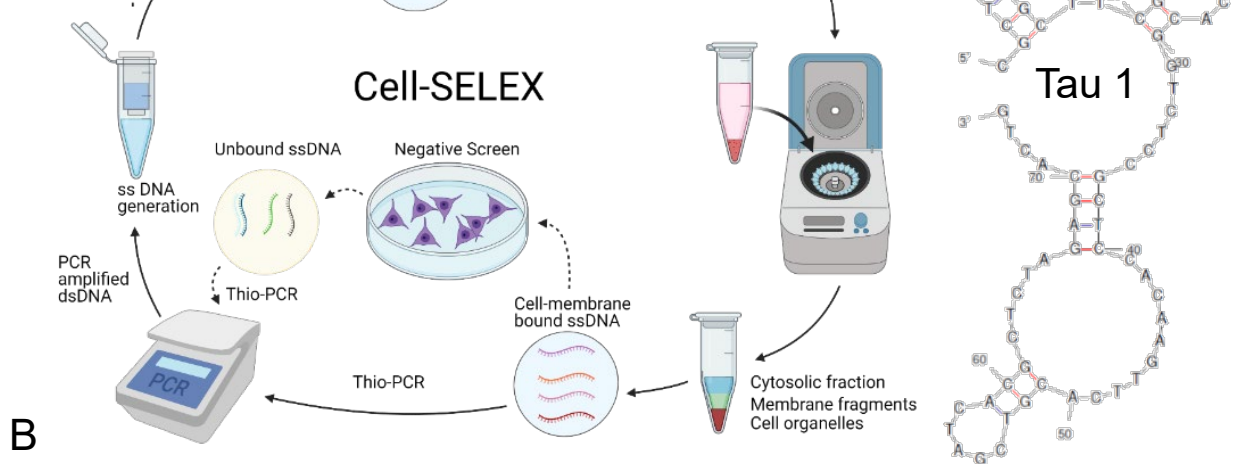

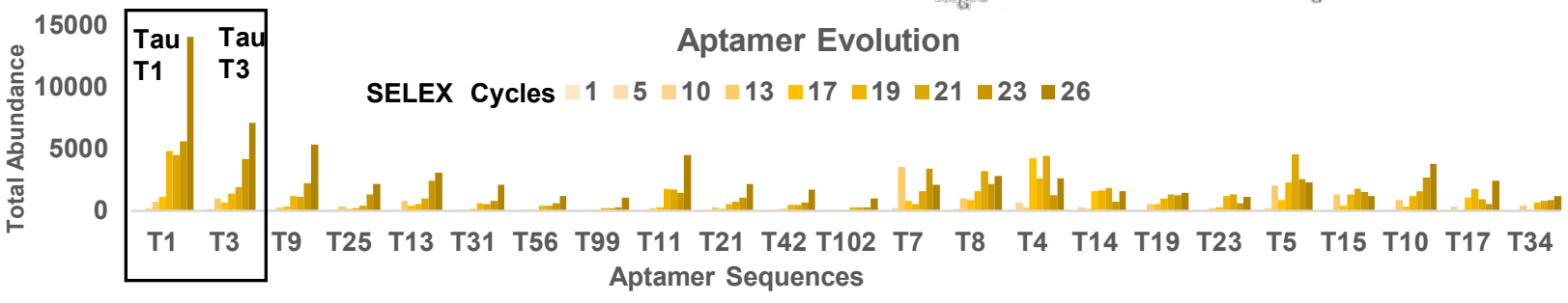

C

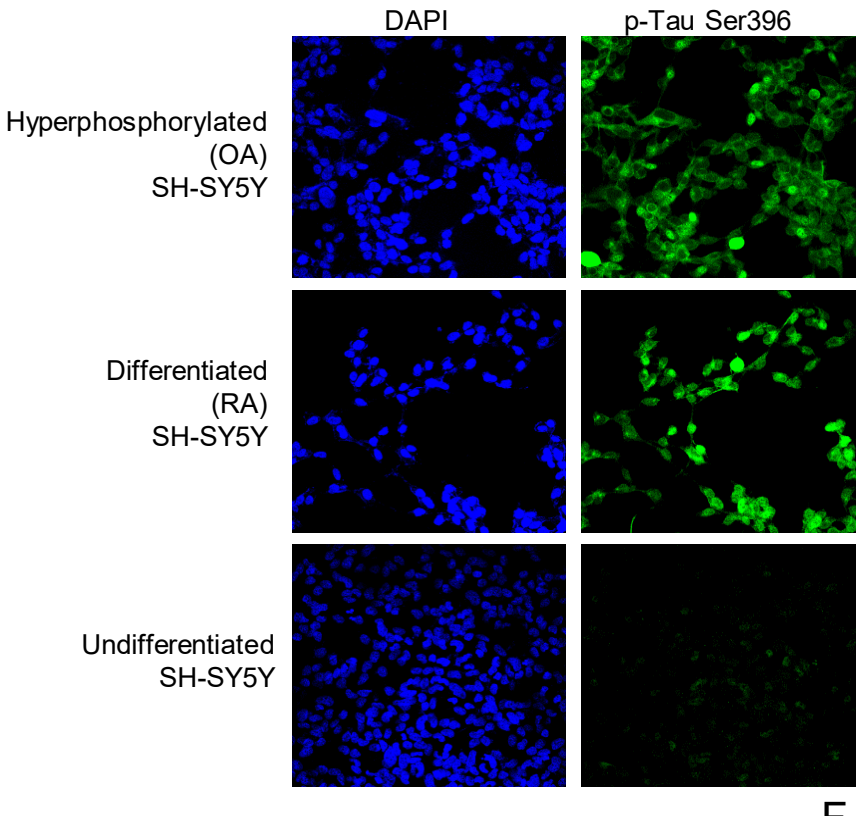

Aptamer Tau1
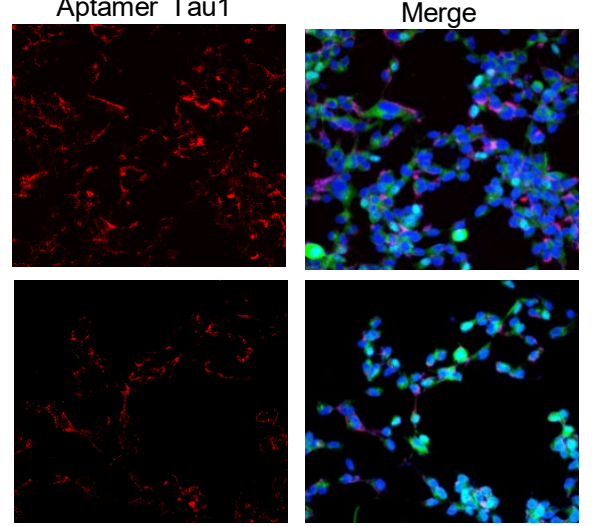

E

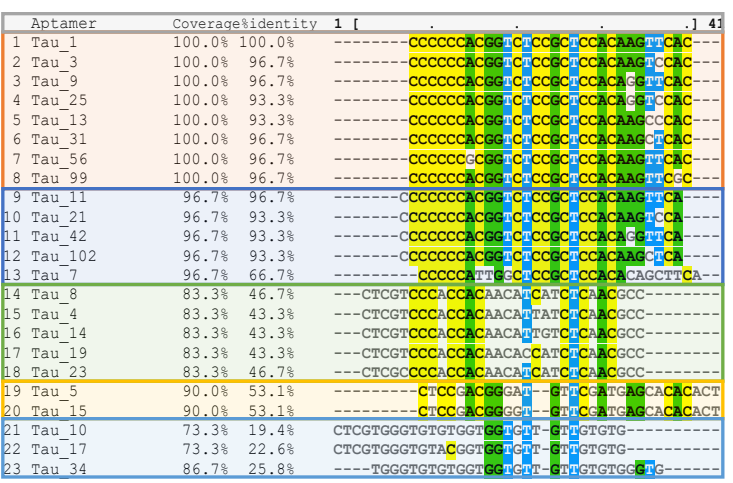
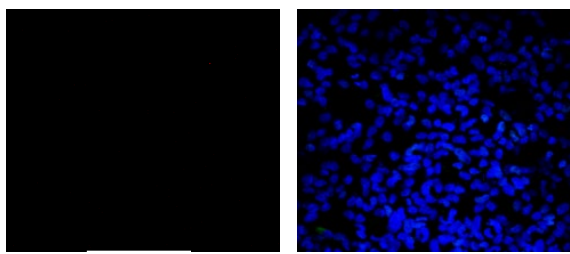

F

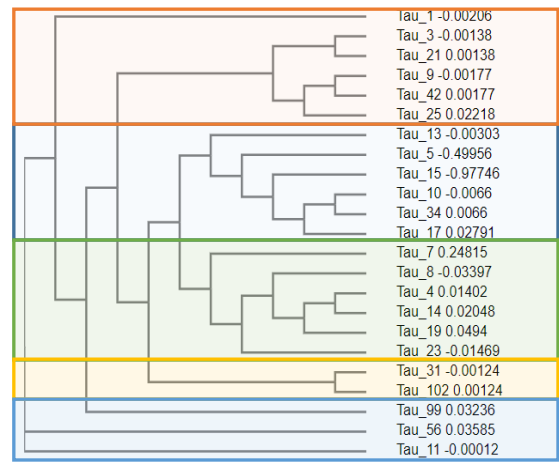


Figure 2: Cell-SELEX to identify biomarkers onset of AD. Okadaic acid treated differentiated SH-SY5Y cells were used as a surrogate for hyperphosphorylative neurons to screen DNA aptamers that specifically recognize the differences between the surfaces of treated and untreated cells, using the cell-SELEX methodology modified to capture membrane binding aptamers. (A) Pictorial representation of the cell-SELEX process (B) Abundance of the top 23 sequences from SELEX cycle $1-26$ depicting their evolution. Note that the fractions are low until about cycle 10, when they increase sharply. Note that the abundance of Tau 1, Tau 3 continually increase with increasing cycle number. (C) Undifferentiated, differentiated (RA) and hyperphosphorylated (OA) SH-SY5Y cells stained with $50 \mathrm{nM}$ Cy5 labelled Tau 1 aptamer for $2 \mathrm{~h}$ at $4^{\circ} \mathrm{C}$. (D) i) Tau 1 and ii) Tau 3 secondary structure using Mfold. (E) Multiple sequence alignment of the top 23 aptamer sequences by MAFFT. Note the existence of five families. $(F)$ Cladogram showing relationship between the Tau aptamer sequences and the aptamer families. 


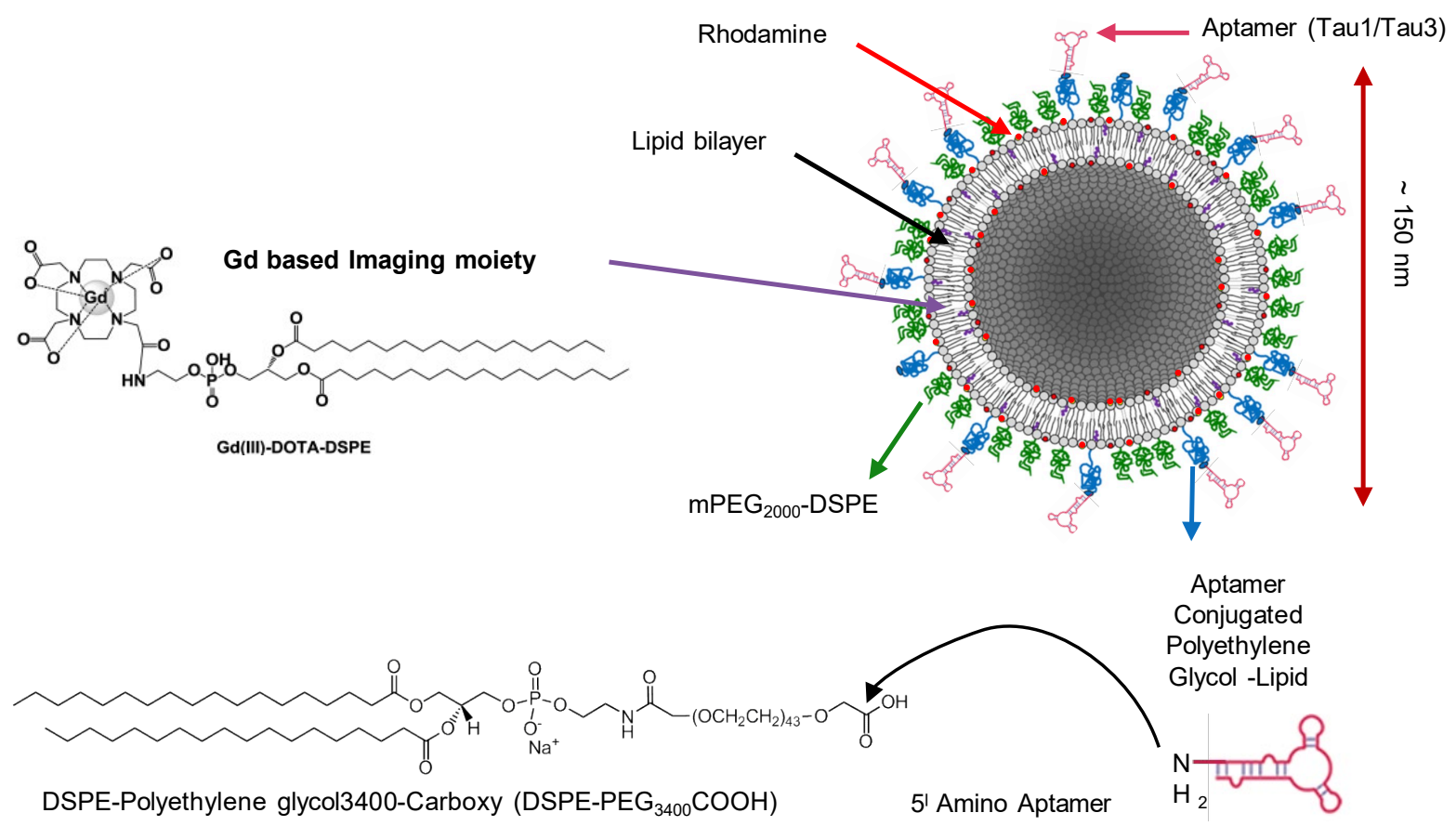

Figure 3: Aptamer-targeted liposomal-Gd nanoparticle TauX contrast agent. The liposomal bilayer incorporates DSPE-DOTA-Gd for MR contrast, DSPE-mPEG2000 to enhance circulation half-life, DSPEPEG3400-aptamer (Tau1/Tau3) for targeting, and lissamine rhodamine for fluorescence imaging. 


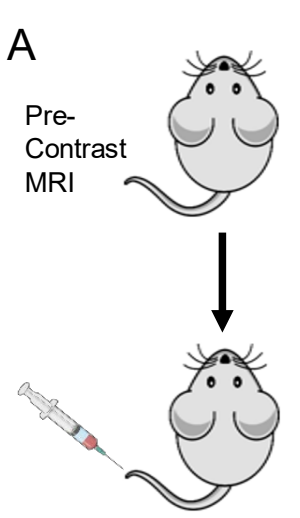

TauX Vein Injection

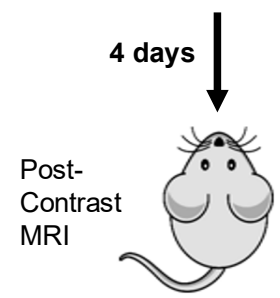

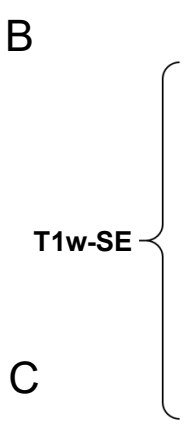
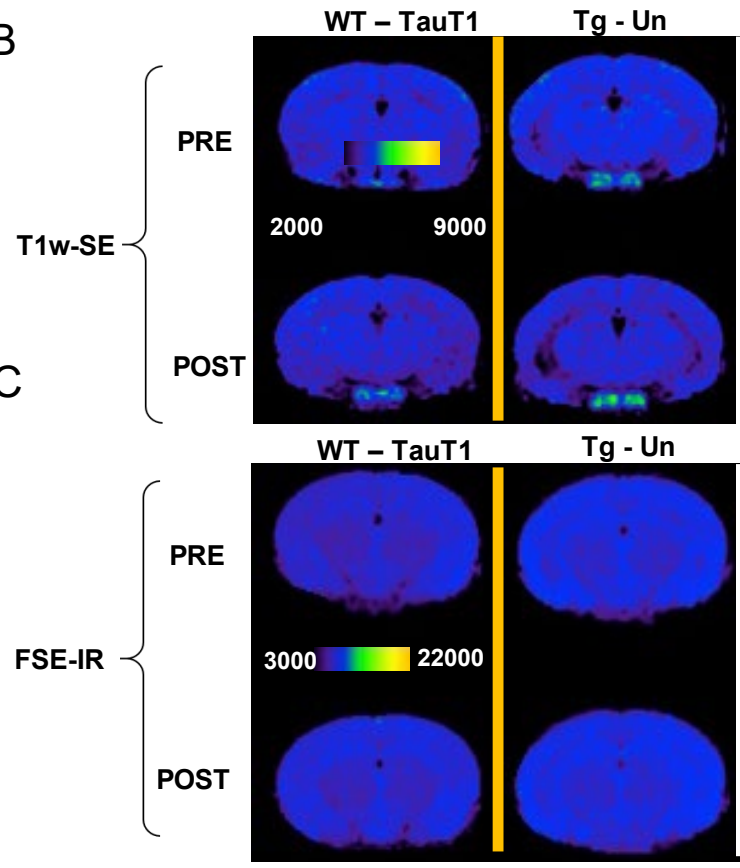

F

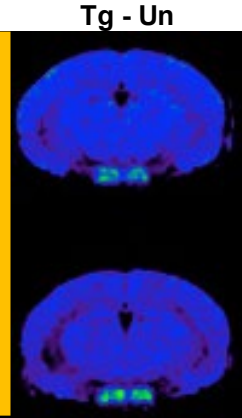

$\mathrm{Tg}$ - Un
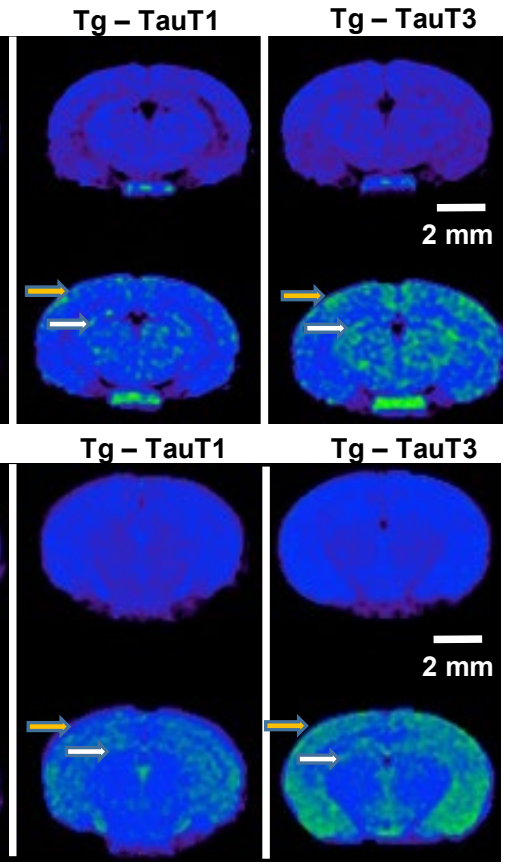

$\mathrm{Tg}-\mathrm{TauT3}$

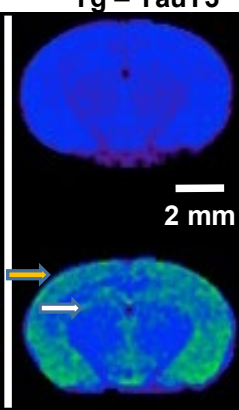

$2 \mathrm{~mm}$
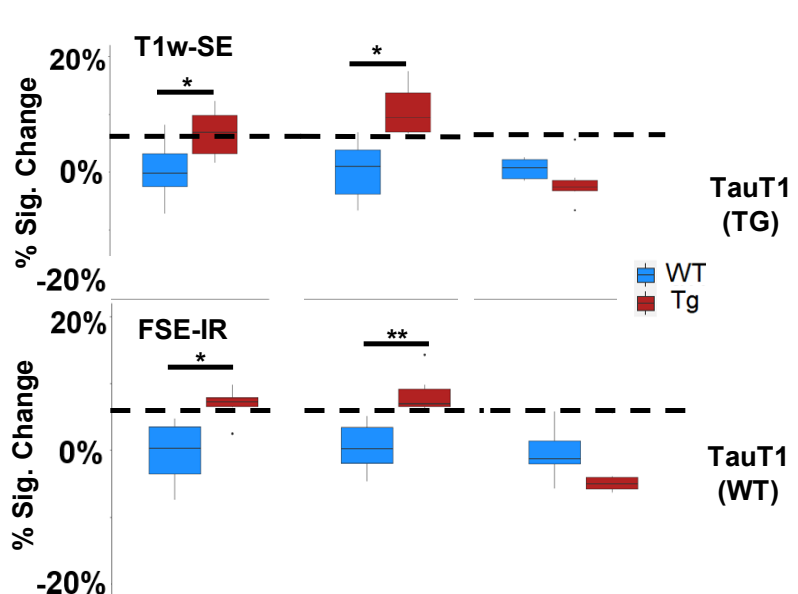

$$
\text { Tau1 } \frac{\text { Tau3 }}{\text { TL }}
$$

E
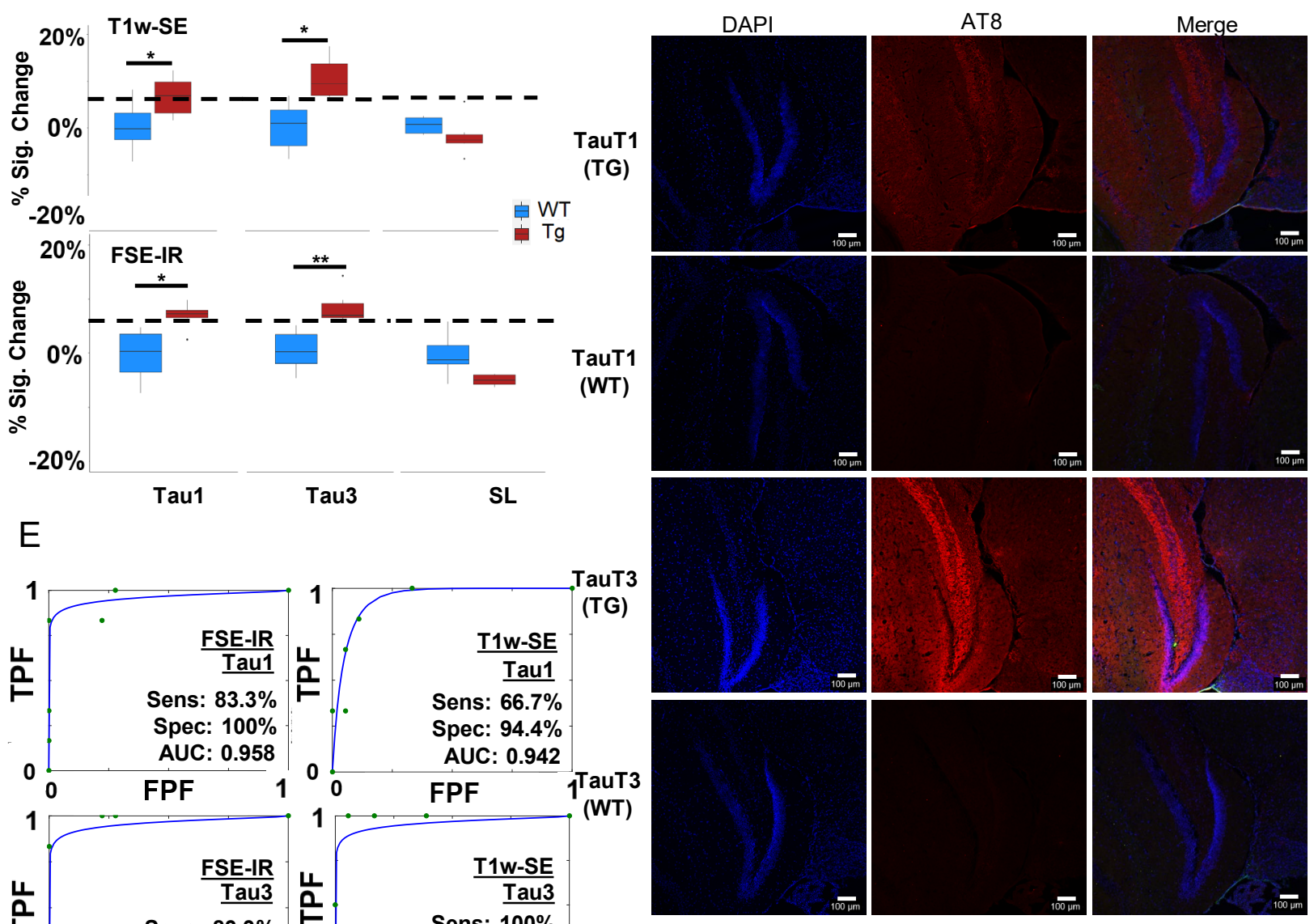

Sens: $100 \%$

Spec: $94.4 \%$ AUC: 0.958

FPF 1 
Figure 4: Magnetic resonance imaging (MRI) and immunofluorescent staining demonstrate signal enhancement as TauX binds to hyperphosphorylated tau in vivo. (A) Two month old mice were injected with $0.17 \mathrm{mmol} \mathrm{Gd} / \mathrm{kg}$ dose TauX. Pre- and post-contrast images for (B)T1-weighted spin echo (T1w-SE) and (C) fast spin echo inversion recovery (FSE-IR) demonstrate signal enhancement in delayed post-contrast scans of transgenic (Tg) P301S mice treated with TauX relative to age-matched wild type (WT) controls. Tg animal showed high enhancement in cortical (yellow arrow) and hippocampal regions (white arrow). Transgenic animal shows no signal enhancement four days after injection of untargeted contrast (UC). Scale bar represents $2 \mathrm{~mm}$. All animals are shown on the same color scale. (D) Box and whisker plots demonstrate signal enhancement in TG animals relative to WT counterparts and UC-treated Tg animals for both T1W-SE and FSE-IR sequences $\left({ }^{*} p<0.05\right.$; $\left.{ }^{* *} p<0.005\right)$. Dotted line indicates signal threshold for determining sensitivity (2 standard deviations above baseline noise, $\sim 6 \%$ ). (E) Receiver operating characteristic (ROC) curves plotting true positive fraction (TPF) against false positive fraction (FPF) demonstrate TauX accuracy in identifying early age $\mathrm{Tg}$ animals. A fitted curve (blue) connects the observed operating points. Area under curve (AUC) is calculated using the fitted curve, and sensitivity (true positive rate) and specificity (true negative rate) for both formulations are listed. (F) The mice selected for TauX injection were genotyped which only revealed the presence of transgene. We confirmed the presence of hyperphosphorylated tau species in the mice by immunofluorescence on the brains sections harvested post MRI scans. Brains were perfused with heparin-PBS and fixed in OCT. IF with antibody AT8 probed for presence of ptau species provided high concordance with genotype confirming the presence of hyperphosphorylative conditions in the mice. 


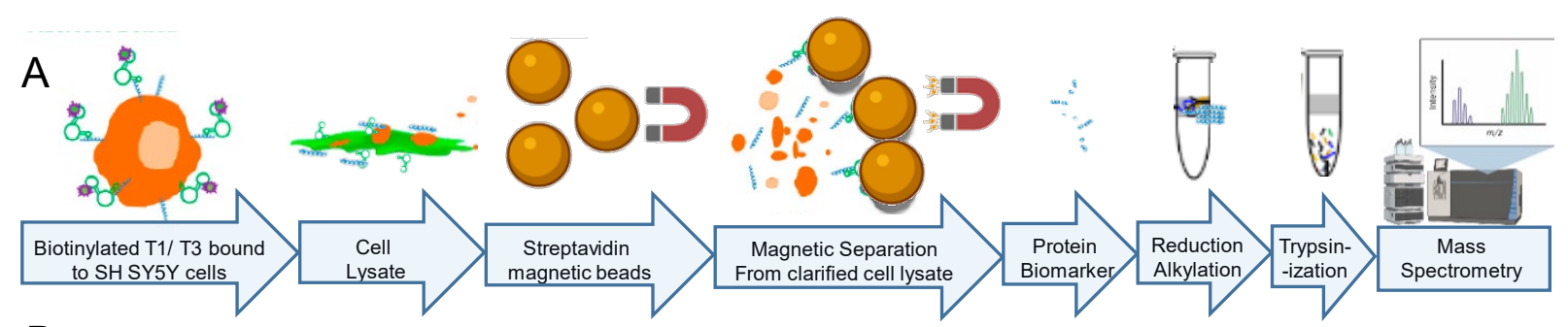

$B$ i)
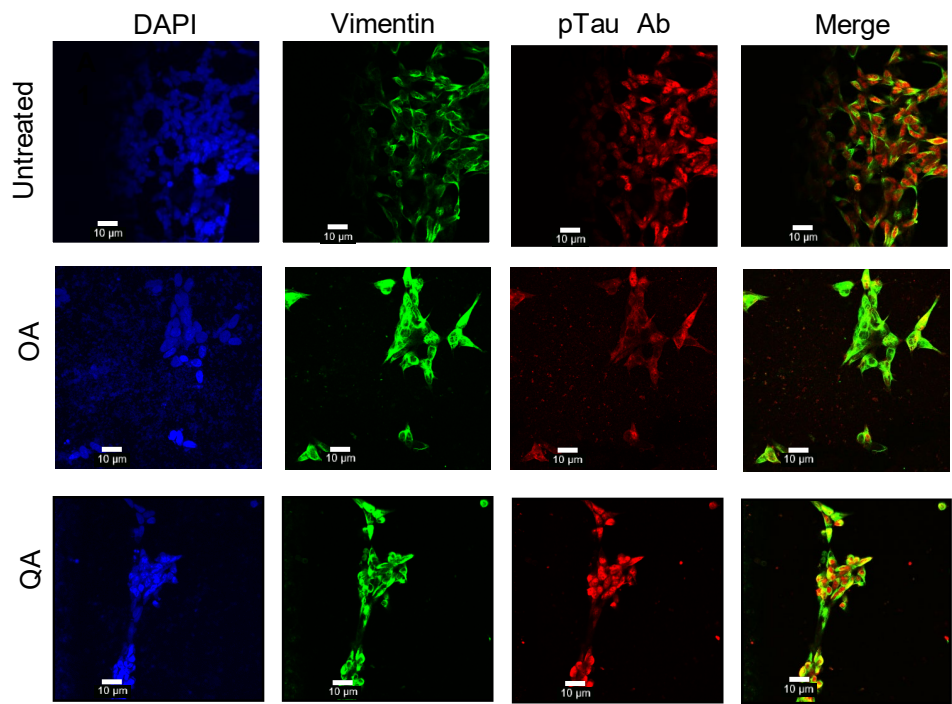

ii)
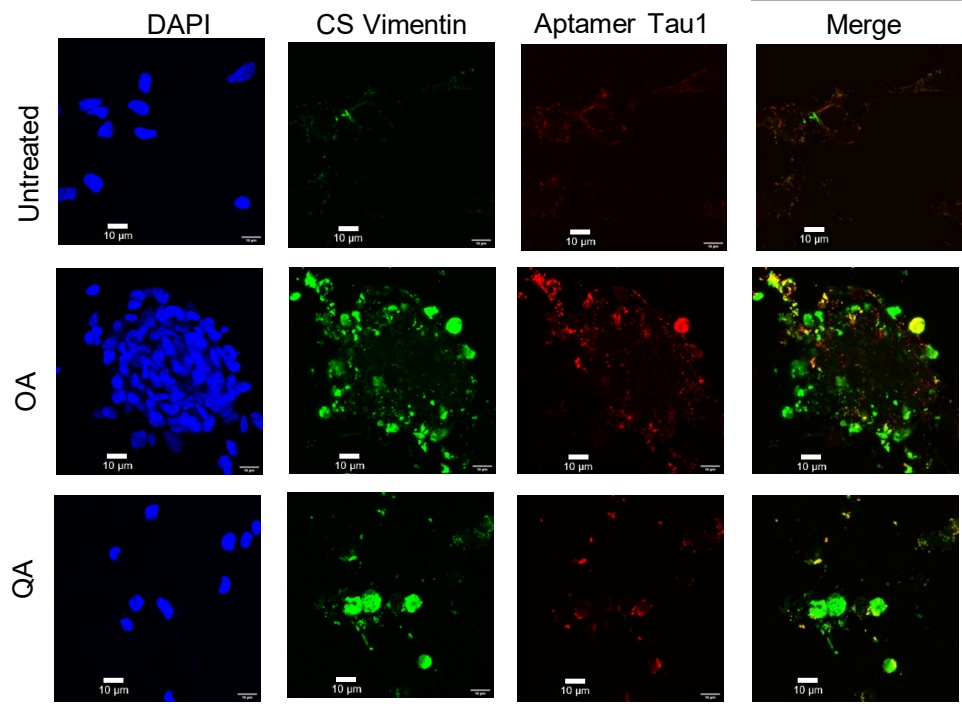

C

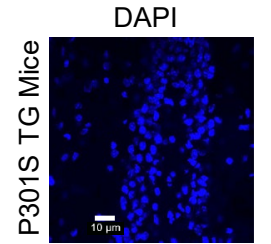

Vimentin
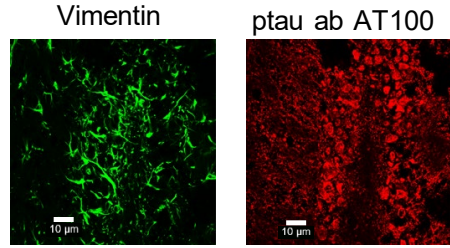

Merge
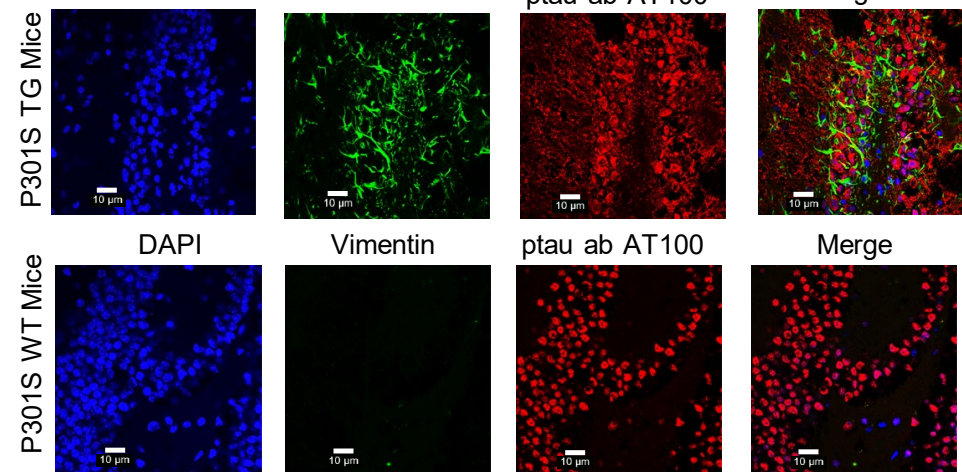

Vimentin

ptau ab AT100

Merge
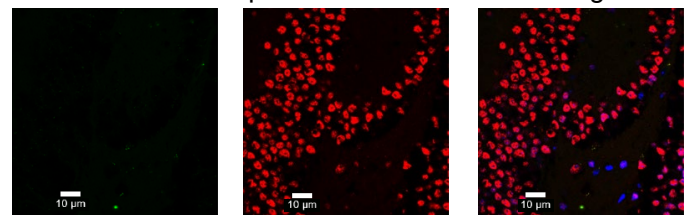
Figure 5: Proteomic analysis for aptamer target identification. A. Tryptic digest of aptamer bound cell membrane analyzed by LC/MS/MS. The raw data files were processed and searched against the SwissProt 2012 01 (Human) database. Vimentin was identified as possible target. B. Presence of the aptamers binding target on SH SY5Y cells; undifferentiated and hyperphosphorylated OA (24h, 30nM) and QA (24h, 100nM); i) co-stained with Vimentin (D21H3) and ptau (AT100) antibodies or ii) cell surface (CS) vimentin (Clone 84-1) antibody and aptamer T1 (50nM); nuclei counterstained with DAPI. C. Expression of Vimentin in P301S TG and WT frozen mouse tissue sections stained with Vimentin (SP20) and ptau (AT100) antibodies and DAPI stained nuclei. 


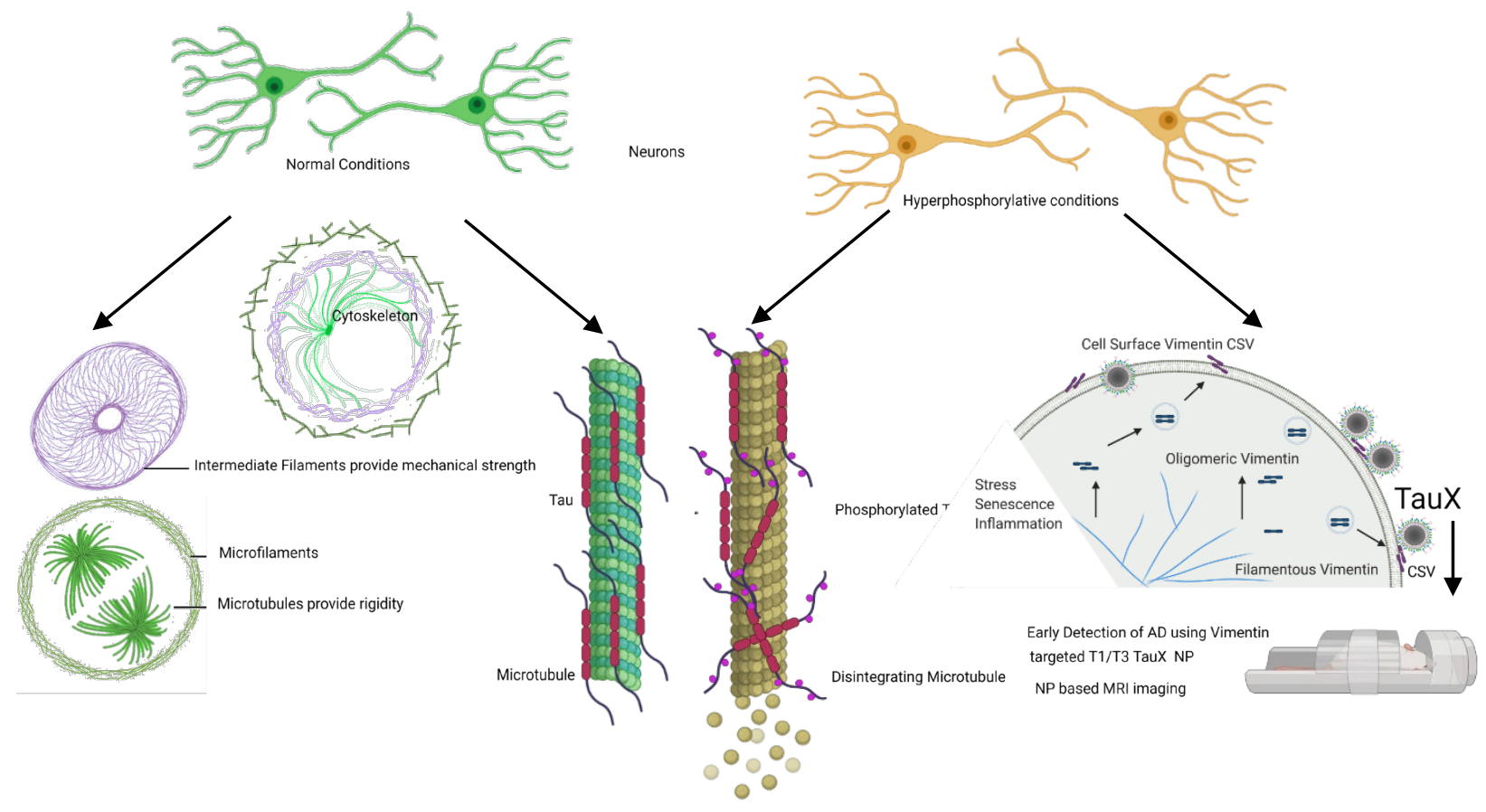

Graphical Abstract: Early detection of Tau pathology. Hyperphosphorylative conditions provoke neuronal cells to undergo changes including on the cell surface. Translocation of Vimentin to the cell surface was exploited to generate an aptamer that was developed for in vivo use as targeted liposomal nanoparticle TauX for early MRI detection of tau pathology in a mouse model 
Supplementary Table 1: Target Identification using aptamer immunoprecipitation and mass spectrometry. Aptamer based immunoprepcipation was performed. Mass-Spectrometric analysis of the trypsin digested aptamer-bound complex was analyzed by LC/MS/MS on an LTQ-Orbitrap-XLmass spectrometer with an Nanoflex system. The raw data files were processed and searched against the SwissProt_2012_01 (Human) database using the Mascot search engine. Exponentially modified protein abundance index (emPAl) are reported in the table below that report protein content proportional to protein content in a mixture.

\section{T1 emPAl Target ID Aptamer Tau1}

$6.3260 \mathrm{kDa}$ heat shock protein, mitochondrial OS=Homo sapiens GN=HSPD1 PE=1 SV=2

3.49Keratin, type II cytoskeletal $1 \mathrm{OS}=$ Homo sapiens $\mathrm{GN}=\mathrm{KRT} 1 \mathrm{PE}=1 \mathrm{SV}=6$

1.29Keratin, type II cytoskeletal 2 epidermal $O S=H o m o$ sapiens $G N=K R T 2 P E=1 S V=2$

0.38Keratin, type II cytoskeletal $6 B$ OS=Homo sapiens $G N=K R T 6 B \quad P E=1 \quad S V=5$

0.38 Keratin, type II cytoskeletal $6 \mathrm{~A}$ OS=Homo sapiens $\mathrm{GN}=\mathrm{KRT} 6 \mathrm{~A} P E=1 \mathrm{SV}=3$

0.11 Keratin, type II cytoskeletal $5 \mathrm{OS}=$ Homo sapiens $\mathrm{GN}=\mathrm{KRT} 5 \mathrm{PE}=1 \mathrm{SV}=3$

4.67Keratin, type I cytoskeletal $10 \mathrm{OS}=$ Homo sapiens $\mathrm{GN}=\mathrm{KRT} 10 \mathrm{PE}=1 \mathrm{SV}=6$

1.66Keratin, type I cytoskeletal 9 OS=Homo sapiens $G N=K R T 9 P E=1 S V=3$

0.97 Keratin, type I cytoskeletal $14 \mathrm{OS}=$ Homo sapiens $\mathrm{GN}=\mathrm{KRT} 14 \mathrm{PE}=1 \mathrm{SV}=4$

0.69Keratin, type I cytoskeletal $17 \mathrm{OS}=$ Homo sapiens $\mathrm{GN}=\mathrm{KRT} 17 \mathrm{PE}=1 \mathrm{SV}=2$

5.01 Single-stranded DNA-binding protein, mitochondrial $O S=$ Homo sapiens $G N=S S B P 1 P E=1 \quad S V=1$

2.35Actin, cytoplasmic $1 \mathrm{OS}=\mathrm{Homo}$ sapiens $\mathrm{GN}=\mathrm{ACTB} \mathrm{PE}=1 \mathrm{SV}=1$

0.13 Vimentin OS=Homo sapiens $\mathrm{GN}=\mathrm{VIM} P E=1 \mathrm{SV}=4$

3.640S ribosomal protein S5 (Fragment) OS=Homo sapiens GN=RPS5 PE=1 SV=1

0.2 Microtubule-associated protein 1B OS=Homo sapiens $\mathrm{GN}=\mathrm{MAP} 1 \mathrm{~B} P E=1 \mathrm{SV}=2$

1.27 Elongation factor 1-alpha $1 \mathrm{OS}=\mathrm{Homo}$ sapiens $\mathrm{GN}=\mathrm{EEF} 1 \mathrm{~A} 1 \mathrm{PE}=1 \mathrm{SV}=1$

1.07Cofilin 1 (Non-muscle), isoform CRA_a OS=Homo sapiens GN=CFL1 PE=1 SV=1

0.28 Leucine-rich PPR motif-containing protein, mitochondrial OS=Homo sapiens GN=LRPPRC PE=1 SV=3

2.74Peptidyl-prolyl cis-trans isomerase $B$ OS=Homo sapiens $G N=P P I B P E=1 \quad S V=2$

2.35Alpha-enolase $\mathrm{OS}=\mathrm{Homo}$ sapiens $\mathrm{GN}=\mathrm{ENO} 1 \mathrm{PE}=1 \mathrm{SV}=2$

0.61 Stress -70 protein, mitochondrial OS=Homo sapiens $\mathrm{GN}=\mathrm{HSPA} 9 \mathrm{PE}=1 \mathrm{SV}=2$

1.91 ATP synthase subunit $O$, mitochondrial OS=Homo sapiens $G N=A T P 5 O P E=1 \quad S V=1$

0.73 Serpin $\mathrm{H} 1 \mathrm{OS}=\mathrm{Homo}$ sapiens GN=SERPINH1 PE=1 SV=2

$0.53 \mathrm{ATP}$ synthase subunit alpha, mitochondrial OS=Homo sapiens $\mathrm{GN}=\mathrm{ATP} 5 \mathrm{~A} 1 \mathrm{PE}=1 \mathrm{SV}=1$

$0.61 \mathrm{ATP}$ synthase subunit gamma, mitochondrial OS=Homo sapiens $\mathrm{GN}=\mathrm{ATP} 5 \mathrm{C} 1 \mathrm{PE}=1 \mathrm{SV}=1$

0.94 Tubulin beta chain OS=Homo sapiens $\mathrm{GN}=\mathrm{TUBB} P E=1 \mathrm{SV}=1$

0.97 Nascent polypeptide-associated complex subunit alpha (Fragment) OS=Homo sapiens $\mathrm{GN}=\mathrm{NACA} P E=1 \mathrm{SV}=1$

$0.2578 \mathrm{kDa}$ glucose-regulated protein OS=Homo sapiens $\mathrm{GN}=\mathrm{HSPA} 5 \mathrm{PE}=1 \mathrm{SV}=2$

T3 emPAl Target ID Aptamer Tau3

4.45Keratin, type II cytoskeletal $1 \mathrm{OS}=$ Homo sapiens $\mathrm{GN}=\mathrm{KRT} 1 \mathrm{PE}=1 \mathrm{SV}=6$

2.92Keratin, type II cytoskeletal 2 epidermal OS=Homo sapiens $\mathrm{GN}=\mathrm{KRT} 2 \mathrm{PE}=1 \mathrm{SV}=2$

2.7Vimentin OS=Homo sapiens GN=VIM PE=1 SV=4

0.43 Keratin, type II cytoskeletal $5 \mathrm{OS}=$ Homo sapiens $\mathrm{GN}=\mathrm{KRT} 5 \mathrm{PE}=1 \mathrm{SV}=3$

5.32Keratin, type I cytoskeletal $10 \mathrm{OS}=$ Homo sapiens $\mathrm{GN}=\mathrm{KRT} 10 \mathrm{PE}=1 \mathrm{SV}=6$

0.45 Keratin, type I cytoskeletal $14 \mathrm{OS}=$ Homo sapiens $\mathrm{GN}=\mathrm{KRT} 14 \mathrm{PE}=1 \mathrm{SV}=4$

1.81T-cell receptor alpha joining 56 (Fragment) OS=Homo sapiens $\mathrm{GN}=T$ RAJ56 PE=4 SV=1

2.62Actin, cytoplasmic $1 \mathrm{OS}=\mathrm{Homo}$ sapiens $\mathrm{GN}=\mathrm{ACTB} P \mathrm{PE}=1 \mathrm{SV}=1$

2.35Actin, cytoplasmic $2 \mathrm{OS}=$ Homo sapiens $\mathrm{GN}=\mathrm{ACTG} 1 \mathrm{PE}=1 \mathrm{SV}=1$

0.46 Actin, aortic smooth muscle OS=Homo sapiens $G N=A C T A 2 P E=1 S V=1$

0.37 Serum albumin $\mathrm{OS}=\mathrm{Homo}$ sapiens $\mathrm{GN}=\mathrm{ALB} P E=1 \mathrm{SV}=1$

1.16Keratin, type I cytoskeletal 9 OS=Homo sapiens $G N=K R T 9 P E=1 S V=3$

$0.6960 \mathrm{kDa}$ heat shock protein, mitochondrial OS=Homo sapiens $\mathrm{GN}=\mathrm{HSPD} 1 \mathrm{PE}=1 \mathrm{SV}=2$

0.12 Myosin-9 OS=Homo sapiens GN=MYH9 PE=1 SV=4

0.1 Isoform 2 of Myosin-10 OS=Homo sapiens GN=MYH10

0.72 Nascent polypeptide-associated complex subunit alpha (Fragment) OS=Homo sapiens GN=NACA PE=1 SV=1

0.1 Nestin OS=Homo sapiens GN=NES PE=1 SV=2

0.71 Single-stranded DNA-binding protein, mitochondrial $O S=$ Homo sapiens $G N=S S B P 1 P E=1 \mathrm{SV}=1$

0.2340 S ribosomal protein $\mathrm{S} 12 \mathrm{OS}=$ Homo sapiens $\mathrm{GN}=\mathrm{RPS} 12 \mathrm{PE}=1 \mathrm{SV}=3$

0.7 Serpin H1 (Fragment) OS=Homo sapiens $\mathrm{GN}=\mathrm{SERPINH} 1 \mathrm{PE}=1 \mathrm{SV}=2$

0.43Transcription factor BTF3 homolog $4 \mathrm{OS}=$ Homo sapiens $\mathrm{GN}=\mathrm{BTF} 3 \mathrm{~L} 4 \mathrm{PE}=1 \mathrm{SV}=1$

0.29 Reticulocalbin-2 OS=Homo sapiens $\mathrm{GN}=\mathrm{RCN} 2 \mathrm{PE}=1 \mathrm{SV}=1$

0.26 Prelamin-A/C OS=Homo sapiens $\mathrm{GN}=\mathrm{LMNA} P E=1 \mathrm{SV}=1$

$0.12 \mathrm{THO}$ complex subunit $4 \mathrm{OS}=$ Homo sapiens $\mathrm{GN}=\mathrm{ALYREF} P E=1 \mathrm{SV}=1$

0.16 Nuclease-sensitive element-binding protein 1 (Fragment) $\mathrm{OS}=$ Homo sapiens $\mathrm{GN}=\mathrm{YBX} 1 \mathrm{PE}=1 \mathrm{SV}=1$

0.3 Peptidyl-prolyl cis-trans isomerase $B$ OS=Homo sapiens $\mathrm{GN}=\mathrm{PPIB} P E=1 \mathrm{SV}=2$

0.1 Isoform 2 of Heat shock protein HSP 90-alpha OS=Homo sapiens GN=HSP90AA1

0.12 Heat shock protein HSP 90-beta OS=Homo sapiens $\mathrm{GN}=\mathrm{HSP} 90 \mathrm{AB} 1 \mathrm{PE}=1 \mathrm{SV}=4$ 
Supplementary Figure 1: Hyperphosphorylative conditions. SH SY5Y cells differentiated with $30 \mu \mathrm{M}$ Retinoic acid for 10 days were exposed to $30 \mathrm{nM}$ Okadaic acid for $24 \mathrm{hrs}$. Under these hyperphosphorylative conditions tau is overexpressed and hyperphosphorylated. Figure shows the presence of p-tau 396 in SH SY5Y cells in hyperphosphorylative conditions..
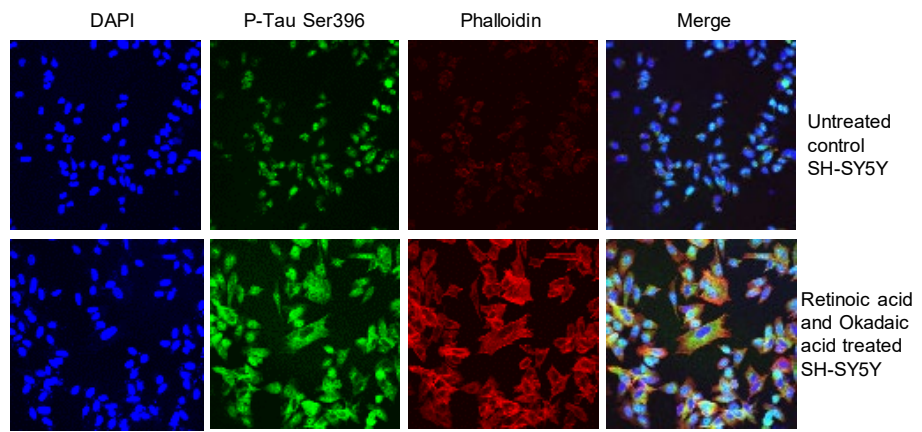
Supplementary Figure 2: Apparent dissociation constants of Aptamer Tau1 and Tau3Target. SH SY5Y and Ren cells were grown in 96-well plates, Retinoic acid was used for differentiation. Okadaic acid for SH SY5Y and quinolinic acid for ReN cells was used to generate hyperphosphorylative conditions. Saturation binding curves were generated using Cy5 labelled Tau1 and Tau3 using a microplate fluorescent reader. The binding constant $\mathrm{Kd}$ apparent was calculated using the inbuilt non-linear regression module using the equation $Y=B m^{*} X /(K d+X)$ in Graph Pad Prism. * error bars smaller than symbol not visualized
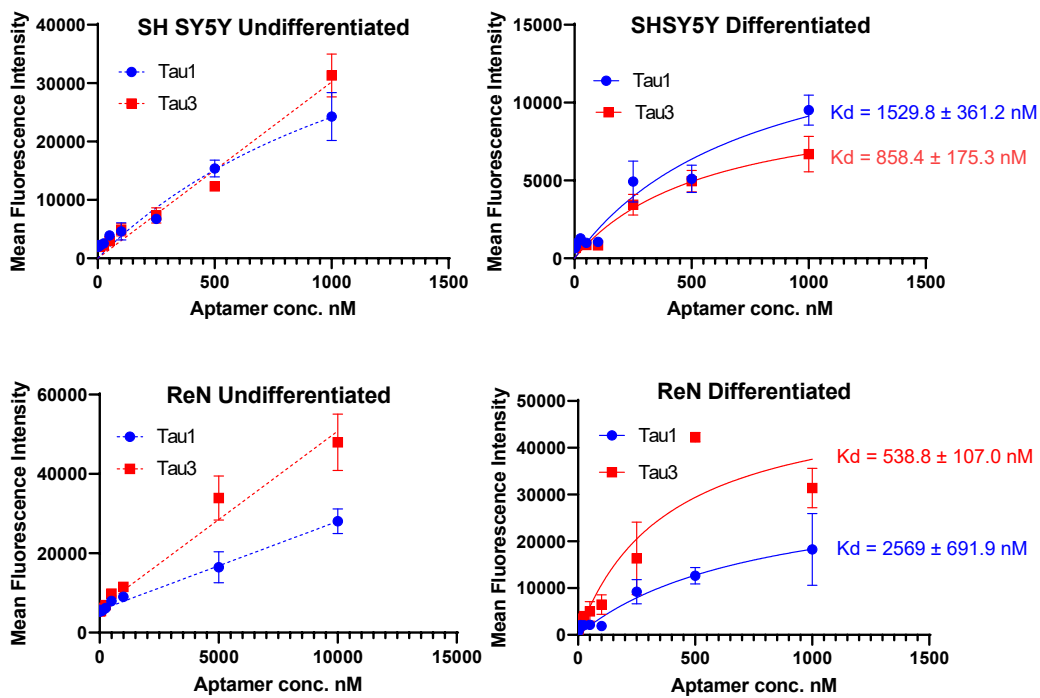
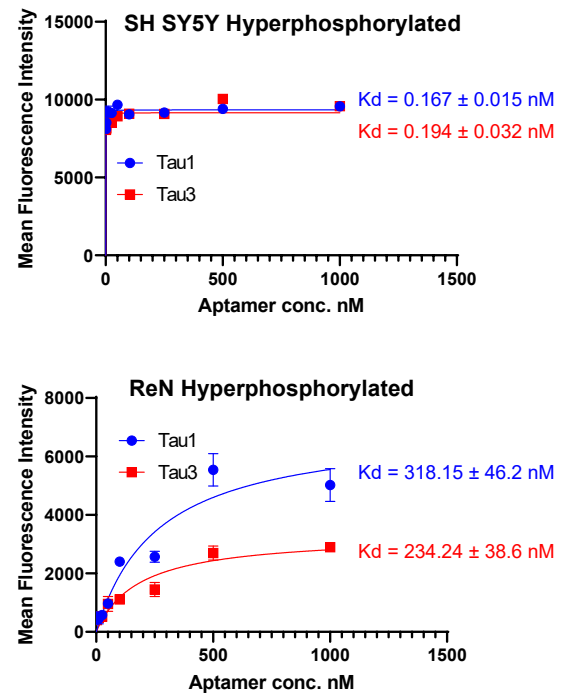